\title{
Nonlinear Aeroelastic Effects in Flapping Wing Micro Air Vehicles
}

\author{
A. Gogulapati * \\ P. P. Friedmann ${ }^{\dagger}$ \\ W. Shyy $\ddagger$ \\ Department of Aerospace Engineering, The University of Michigan, Ann Arbor, 48109, USA
}

\begin{abstract}
A nonlinear aeroelastic model for flapping micro air vehicle wings undergoing prescribed rigid body motion and moderate-to-large flexible deformation is presented. The aeroelastic model is obtained by coupling a nonlinear structural dynamic model based on the MARC code with approximate unsteady aerodynamic loads. The aeroelastic response is obtained using an updated Lagrangian method. The aerodynamic model is based on potential flow that uses a combined circulation/vorticity approach to compute the unsteady aerodynamic loads. An important ingredient of the aerodynamic model is the inclusion of a leading edge separation and subsequent vortex formation. The airfoil is modeled by bound vorticity and the wake is represented by point vortices. The unsteady loads computed using this approach are used to examine the effect of wing flexibility on the response. Preliminary aeroelastic response results indicate that, for the parameters considered, the effect of aerodynamic loads is relatively minor compared to the effect of inertial loads; furthermore, wing flexibility may have a favorable effect on lift generation.
\end{abstract}

\section{Nomenclature}

$\begin{array}{ll}a & \text { Distance between mid-chord and pitch axis, measured along } X^{A} \\ A & \text { Unrotated or global coordinate system (or the reference frame) } \\ A_{1}-A_{11} & \text { Coefficients that depend on the airfoil degrees of freedom } \\ B & \text { Rotated coordinate system } \\ \mathbf{B}_{L}, \mathbf{B}_{N L} & \text { Transformation matrices that are derived from shape function matrices } \\ b & \text { Airfoil semi-chord } \\ c & \text { Chord } \\ \mathbf{C} & \text { Matrix or tensor of material constants } \\ C_{D} & \text { Drag coefficient } \\ C_{L} & \text { Lift coefficient } \\ C_{M} & \text { Moment coefficient } \\ e_{i j} & \text { Cartesian components of the Cauchy strain tensor } \\ E & \text { Young's modulus } \\ \mathbf{F} & \text { Force vector obtained from stress in the equations of motion } \\ F_{i n e r t i a l} & \text { Virtual work done by inertial forces } \\ \hat{F} & \text { Aerodynamic force measured in a non-rotating frame } \\ f & \text { Flapping frequency } \\ h & \text { Plunge degree of freedom } \\ \bar{h} & \text { Non-dimensional plunge amplitude }=h / c \\ \hat{I} & \text { Impulse of the vortices in the airfoil-wake system } \\ \hat{I}_{m} & \text { Moment of the impulse of the vortices in the airfoil-wake system } \\ i, j, k, r, s & \text { Indices that represent components of vectors or tensors } \\ \imath & \sqrt{-1} \\ k & \text { Reduced frequency }=\pi f c / U_{\infty}\end{array}$

${ }^{*}$ Ph.D. candidate, Student Member AIAA.

${ }^{\dagger}$ FXB Professor, Fellow AIAA and AHS.

‡Clarence "Kelly" Johnson Professor, Fellow AIAA. 


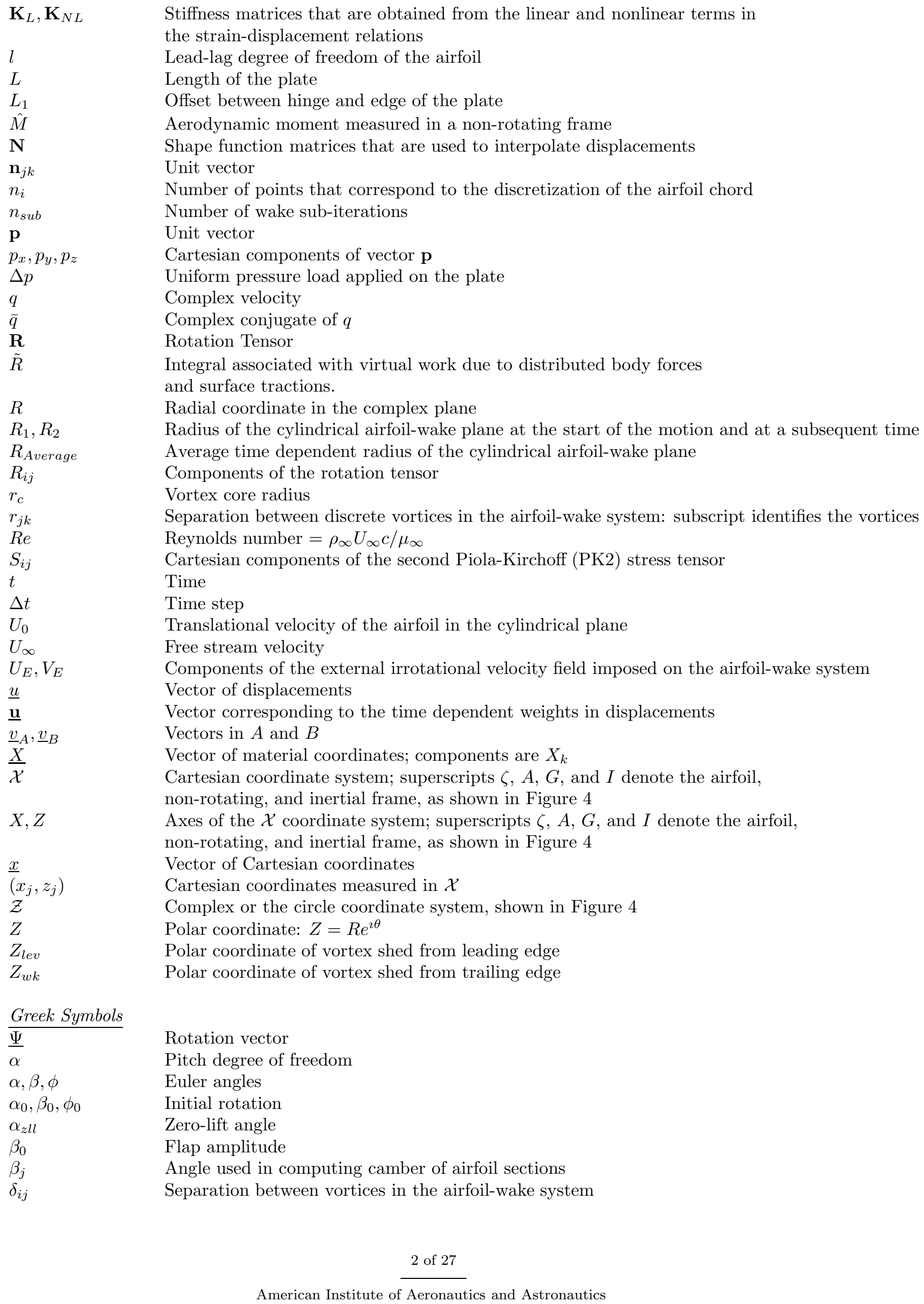




\begin{tabular}{|c|c|}
\hline $\begin{array}{l}\delta_{l} \\
\delta_{p}\end{array}$ & $\begin{array}{l}\text { Displacement of point on the edge measured along the length of the rigid plat } \\
\text { Displacement of point on the edge measured perpendicular to the length } \\
\text { of the rigid plate }\end{array}$ \\
\hline$\delta_{A B}$ & Magnitude of displacement of point on the edge $=\sqrt{\delta_{l}^{2}+\delta_{p}^{2}}$ \\
\hline$\epsilon_{\text {sep }}$ & Non-dimensional parameter in the vortex amalgamation algorithm \\
\hline$\varepsilon$ & $=(\tau-\imath \sigma) / R$ \\
\hline$\varepsilon_{i j}$ & Cartesian components of the Green-Lagrange strain tensor \\
\hline$\gamma$ & Vorticity \\
\hline$\gamma_{b}$ & Total bound vorticity on the airfoil \\
\hline$\gamma_{f s}$ & Component of vorticity due to free stream \\
\hline$\gamma_{l e v}$ & Component of vorticity due to wake from leading edge \\
\hline$\gamma_{q s}$ & Quasi-steady component of vorticity \\
\hline$\gamma_{u s}$ & Vorticity due to motion of airfoil \\
\hline$\gamma_{w i}$ & Wake induced vorticity \\
\hline$\gamma_{w k}$ & Component of vorticity due to wake from trailing edge \\
\hline$\Gamma$ & Circulation \\
\hline$\Gamma_{0}$ & Quasi-steady circulation \\
\hline$\mu_{\infty}$ & Viscosity of the free stream \\
\hline$\nu$ & Poisson's ratio \\
\hline$\omega$ & Frequency \\
\hline$\rho$ & Density of the material \\
\hline$\rho_{\infty}$ & Free stream density of the fluid \\
\hline$\psi$ & Rotation angle \\
\hline$\sigma$ & Camber parameter \\
\hline$\sigma_{i j}$ & Cartesian components of Cauchy stress tensor \\
\hline$\theta$ & Angular coordinate in the complex plane \\
\hline$\theta_{1}, \theta_{2}, \theta_{3}$ & Euler angles that represent successive rotations about body fixed axes \\
\hline$\tau$ & Thickness parameter \\
\hline$(\xi, \eta)$ & Complex coordinates in the airfoil-wake cylindrical plane \\
\hline$\left(\xi_{L E}, \eta_{L E}\right)$ & Complex coordinates of the leading edge of the airfoil \\
\hline$\left(\xi_{T E}, \eta_{T E}\right)$ & Complex coordinates of the trailing edge of the airfoil \\
\hline$\zeta$ & Position vector in the airfoil coordinate system, $\zeta=\xi+\imath \eta$ \\
\hline$\zeta_{\text {latest }}$ & Position vector of the most recent vortex in the airfoil coordinate system \\
\hline$\zeta_{\text {previous }}$ & Position vector of the previously shed vortex in the airfoil coordinate system \\
\hline$\zeta_{\text {edge }}$ & Position vector of the leading or trailing edge of the airfoil \\
\hline & Position vector measured in a non-rotating coordinate system \\
\hline
\end{tabular}

\section{Introduction}

Micro Air Vehicles (MAVs) are flight vehicles whose geometric dimensions are typically $15 \mathrm{~cm}$ or less and weigh typically less than 100 grams. ${ }^{1,2}$ Existing MAVs can be broadly classified into three types: fixed wing, rotary wing, and flapping wing designs. While fixed and rotary wing MAVs benefit from available fixed and rotary wing aircraft technologies, flapping wing MAVs are fundamentally different and are inspired from biological flyers such as bats, small birds, and insects. The principal aspects of these flight vehicles have been described in Refs. [1,2].

Flapping wing micro air vehicles $\left(\right.$ FWMAVs) that operate at low Reynolds number $\left(10^{2}<\operatorname{Re}<10^{5}\right)$ and low velocities of forward flight $(<15 \mathrm{~m} / \mathrm{s})$ have received considerable attention. A significant portion of research on flapping wing vehicles has focused on understanding the mechanisms that generate unsteady aerodynamic forces; the work by Ellington ${ }^{3}$ was one of the earliest. Subsequent research, which has been reviewed in several excellent articles ${ }^{4-9}$ and books, ${ }^{1,10}$ spans a broad range of experimental, computational, and semi-empirical studies and includes contributions from biologists, fluid mechanicians and engineers.

Attempts to model the aerodynamic environment in a quantitative manner have been based on two approaches: (1) approximate aerodynamic theories based on potential flow solutions, and (2) computational 
fluid dynamics (CFD) simulations based on Navier Stokes (NS) aerodynamics. Approximate theories are based on a variety of steady, quasi-steady and unsteady assumptions. Historically, simple steady and quasisteady models were used to compute aerodynamic forces generated by biological flapping wing flyers. ${ }^{3}$ These models identified the overall nature of the aerodynamic loading in a qualitative manner and consistently underestimated forces generated by flapping wings. ${ }^{1,6,10}$ Unsteady aerodynamic formulations used for flapping wing problems can be classified as assumed (or prescribed) wake and free wake models. Assumed wake models, developed originally for aeroelastic studies of fixed wing vehicles, are two-dimensional linear thin airfoil theories that are valid for small plunge (bending) and pitch (torsional) displacements. ${ }^{11}$ The role of leading edge vortices (LEVs) in enhancing thrust generated by flapping wings has been recognized, ${ }^{12-14}$ and recent studies have attempted to incorporate the effect of the LEV in assumed wake formulations. ${ }^{15-17}$ These approaches involve modifying the unsteady aerodynamic lift and moment expressions obtained from Theodorsen's theory by using a leading edge suction analogy ${ }^{18}$ that was originally derived for steady separated flow on delta wings for moderate angles of attack (typically less that 40 degrees). Free wake models include unsteady panel methods ${ }^{19,20}$ and discrete vortex methods. ${ }^{21-23}$ These approaches, which account for evolution of the wake, provide a reasonable approximation to the development of the unsteady wake during a flapping cycle. A two-dimensional panel method was used in Ref. [20], and reasonably good agreement was found with CFD simulations for the range of parameters considered. However, LEVs were not accounted for in this study. Two dimensional discrete vortex formulations that account for flow separation have been considered in Refs. [21-23]. The model developed in Ref. [21], which accounts for separation close to the leading edge, compared well with experimental data for airfoils in steady flow. In this approach the chordwise location of the separation point, which may be obtained using independent computations or experiments, is explicitly incorporated into the formulation. Comprehensive treatment of an unsteady aerodynamic model, based on a discrete vortex method, that is applicable to insect-like flapping wings in hover is presented in Refs. [22,23]. The model was used to simulate rigid wings, and for the cases considered, compared well with experimental data on flapping wings. ${ }^{22}$ Studies that have used CFD for generating the aerodynamic loading on rigid and flexible airfoils that are undergoing prescribed pitch and plunge motions ${ }^{20,24,25}$ have identified the presence of leading and trailing edge vortices and found good agreement with wake structures that were observed in experiments.

Insect wings, which are known to undergo moderate-to-large flexible deformation, are composed of veins and membranes, and are extremely diverse in shape, size and configuration. ${ }^{26}$ Structural models of bioinspired wings have employed membranes supported by tubular veins. ${ }^{27,28}$ Early attempts to model flexibility in flapping wings included experimental studies that used geometrically scaled wings and computational studies that used simple physical models. ${ }^{28-30}$ These studies suggested that wing flexibility is an important factor in enhancing the aerodynamic efficiency of a flapping wing. An important finding in Refs. $[29,30]$ was that a dominant component of the loading on flapping wings is due to inertia loads. Recent experimental studies ${ }^{15,16}$ support this conclusion. Therefore, development of an appropriate structural dynamic model is an important aspect in the design of flapping wing MAVs. Previous treatment of wing flexibility using computational approaches has consisted of linear finite element models, ${ }^{15}, 19$ combined with free vibration mode shapes for order reduction. More recently, nonlinear thin-shell models have also been considered. ${ }^{31}$ However, the systematic treatment of moderate-to-large flexible deformations of flapping wings has received limited attention.

The importance of wing flexibility in enhancing the performance of FWMAVs has been mentioned in a number of studies; ${ }^{29,30}$ however, a quantitative substantiation of this claim based on a coupled fluid-structure, or aeroelastic, study has not been carried out. Only a limited number of studies ${ }^{15,19}$ have attempted to address this issue in a systematic manner. In the earliest study, where a linear finite element model of the wing was coupled with an unsteady panel method, ${ }^{19}$ the wing model was based on experimentally obtained geometric and inertial data on Moth wings. More recent computational studies ${ }^{15,16}$ have considered wing models based on membranes reinforced by metal or composite frames. The aerodynamic loads were obtained using Theodorsen's theory modified using Polhamus leading edge suction analogy. ${ }^{18}$ In both studies ${ }^{15,19}$ flexibility was incorporated in a linear manner using free vibration mode shapes of the wing. Reference [19] reported a slight increase in lift due to wing flexibility; however, this study concluded that wing flexibility needs to be considered along with LEVs and wing-wake interaction in order to provide a more complete picture. References $[15,16]$ noted that wing flexibility altered the aerodynamic loads generated due to flapping; therefore, an important conclusion of this study was that the effect of flexibility cannot be ignored when computing aerodynamic loads. 
Typical bio-inspired wings in FWMAVs may consist of tubular beams, plates, and membranes, as shown in Figure 1 and are capable of undergoing large amplitude rigid body motion ${ }^{32}$ as well as moderate-to-large flexible deformation. The aerodynamic loads due to the wing kinematics and flexibility are also complicated. Therefore, the overall goal of this research is to simulate the aeroelastic behavior of FWMAVs using computational tools. The specific objectives of the paper are:

1. To present the development of a nonlinear aeroelastic model based on combining the MARC nonlinear finite element analysis software ${ }^{33}$ with an approximate unsteady aerodynamic model. ${ }^{22,23}$

2. Conduct systematic validation studies to develop confidence in the code.

3. Explore the effect of wing flexibility on the aeroelastic response and unsteady loads generated by typical flapping wings.

\section{Nonlinear Aeroelastic Analysis}

The aeroelastic model developed in this study consists of a nonlinear, finite element based, structural dynamic model combined with unsteady aerodynamic loads computed from approximate theories. The two ingredients of the model together with the approach used to calculate aeroelastic response are described next.

\section{A. Structural Dynamic Model and Wing Kinematics}

The bio-inspired wing, depicted in Figure 1, is modeled using $\mathrm{MARC}^{33}$ and consists of beam, plate, shell, and membrane elements capable of undergoing large rigid body rotations as well as moderate-to-large flexible deformation. The elements are compatible with a variety of material constitutive laws so that isotropic as well as anisotropic structures can be modeled. Wing kinematics, which consist of large amplitude, prescribed, time dependent rigid body rotations, ${ }^{32}$ are applied as boundary conditions at the root of the wing. Finally, user defined subroutines and in-built functions, provided in MARC, are used to extract nodal data at each time step and impose unsteady aerodynamic loads on the wing.

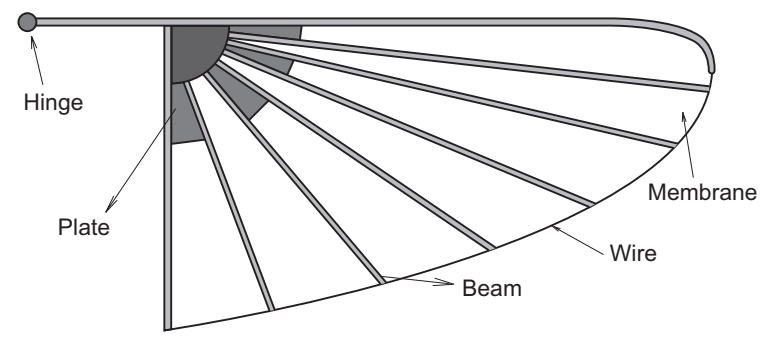

Figure 1. Bio-inspired model of a Hummingbird wing

Large rigid body rotations, also called finite rotations, have received considerable attention in literature. ${ }^{34-36}$ Mathematically, any general rotation in three dimensional Euclidean space may be represented as a proper orthogonal tensor, and the operation of rotation on a vector space is equivalent to multiplication by this tensor. Often, parametric representation of rotations leads to efficient formulation of the dynamics. Such parameterizations are broadly classified as vectorial and non-vectorial. ${ }^{34,36}$ In MARC, rigid body rotations may be imposed as boundary conditions either using an incremental form of the rotation vector ${ }^{33}$ or as displacements at two or more nodes. However, Euler angles have been used in Ref. [32]. Therefore, a brief discussion on the conversion from one form to the other is provided next.

Time dependent rigid body rotations can be input in MARC using an incremental form of the rotation vector that is referred to the unrotated or global coordinate system. ${ }^{37}$ Each rotation increment is converted to the corresponding rotation tensor and applied as a successive rotation. Definitions of the rotation vector and corresponding tensor are given below. The rotation vector is defined as:

$$
\underline{\Psi}(t)=\psi(t) \mathbf{p}(t)
$$


Denote by $A$ and $B$ two coordinate systems that coincide before the rotation, then $\underline{v}_{A}$ and $\underline{v}_{B}$ denote vectors that are fixed in $A$ and $B$ respectively such that $\underline{v}_{A}$ coincides with $\underline{v}_{B}$ before the rotation. When $B$ has rotated with respect to $A, \underline{v}_{A}$ and $\underline{v}_{B}$ are related by

$$
\underline{v}_{A}=\mathbf{R}\left(\underline{\Psi}_{)} \underline{v}_{B}\right.
$$

where $\mathbf{R}(\underline{\Psi})$ is the rotation tensor associated with $\underline{\Psi}$ and is defined by ${ }^{36}$

$$
\mathbf{R}(\underline{\Psi})=\left[\begin{array}{ccc}
c_{\psi}+p_{x}^{2}\left(1-c_{\psi}\right) & -p_{z} s_{\psi}+p_{x} p_{y}\left(1-c_{\psi}\right) & p_{y} s_{\psi}+p_{x} p_{z}\left(1-c_{\psi}\right) \\
p_{z} s_{\psi}+p_{x} p_{y}\left(1-c_{\psi}\right) & c_{\psi}+p_{y}^{2}\left(1-c_{\psi}\right) & -p_{x} s_{\psi}+p_{y} p_{z}\left(1-c_{\psi}\right) \\
p_{y} s_{\psi}+p_{x} p_{z}\left(1-c_{\psi}\right) & p_{x} s_{\psi}+p_{y} p_{z}\left(1-c_{\psi}\right) & c_{\psi}+p_{z}^{2}\left(1-c_{\psi}\right)
\end{array}\right]
$$

where, an abbreviated notation $c_{\psi}=\cos \psi$, and $s_{\psi}=\sin \psi$ is used. From Eq. (3), and noting that $p_{x}^{2}+p_{y}^{2}+p_{z}^{2}=1$, the following relations are obtained:

$$
\begin{aligned}
\psi & =\frac{1}{2} \cos ^{-1}\left[R_{11}+R_{22}+R_{33}-1\right] \\
p_{x} & =\frac{\left(R_{32}-R_{23}\right)}{2 \sin \psi} \\
p_{y} & =\frac{\left(R_{13}-R_{31}\right)}{2 \sin \psi} \\
p_{z} & =\frac{\left(R_{21}-R_{12}\right)}{2 \sin \psi}
\end{aligned}
$$

Thus, given any rotation tensor that relates vectors $\underline{v}_{A}$ and $\underline{v}_{B}$, the corresponding rotation vector may be obtained.

An alternative is to use Euler angles that correspond to body-fixed rotations, wherein successive rotations are performed about non-parallel body-fixed axes. ${ }^{36}$ Rotation using Euler angles is specified using three angles and a sequence of rotation. Using the previous example of coordinate systems $A$ and $B$, and vectors $\underline{v}_{A}$ and $\underline{v}_{B}$, when the three angles in sequence are $\theta_{1}, \theta_{2}$, and $\theta_{3}$, then

$$
\underline{v}_{A}=\mathbf{R}_{\left(\theta_{1}, \theta_{2}, \theta_{3}\right)}^{T} \underline{v}_{B}
$$

where $\mathbf{R}_{\left(\theta_{1}, \theta_{2}, \theta_{3}\right)}$ is the rotation tensor corresponding to the complete rotation and is defined as

$$
\mathbf{R}_{\left(\theta_{1}, \theta_{2}, \theta_{3}\right)}=\mathbf{R}\left(\theta_{3}\right) \mathbf{R}\left(\theta_{2}\right) \mathbf{R}\left(\theta_{1}\right)
$$

Here, each $\mathbf{R}\left(\theta_{\mathbf{i}}\right)$, for $i=1,2,3$, is obtained by substituting the magnitude and the axis of rotation to the $\mathrm{i}^{\text {th }}$ Euler angle into Eq. (3). Note that different sequences of rotation may result in the same rotation tensor; thus, Euler angles that correspond to a given rotation tensor are not unique. ${ }^{36}$

Note that the motion of any point due to a time dependent rotation in which the axis of rotation is fixed corresponds to motion in a plane. Rotations in which both the axis and magnitude of rotation are time dependent correspond to motion in three dimensional space. Therefore, the following terminology is adopted in this document: rotations that result in motion on a plane are referred to as two-dimensional rotations, and those which result in motion in three-dimensional space are referred to as three-dimensional rotations. Thus, wing kinematics, as described in Refs. [31,32], are three-dimensional rotations.

\section{B. Unsteady Aerodynamic Model}

The unsteady aerodynamic loads generated by a flexible wing undergoing prescribed rigid body motion are obtained from an approximate theory that uses a discrete vortex representation of the wake. ${ }^{22,23}$ This approach was initially derived and implemented for rigid wings in Refs. [22, 23]. Accounting for wing flexibility does not require a significant modification of the approach or its implementation. The approach and its implementation for flexible wings are described next. 


\section{Assumptions}

The unsteady aerodynamic model is based on the following assumptions:

(a) The flow field is incompressible, irrotational and inviscid, which implies potential flow; therefore, the velocity potential is obtained from the solution of the Laplace's equation.

(b) The wake associated with the wing has two distinct components: one due to flow separation at the leading edge (LE) and a conventional wake at the trailing edge (TE).

(c) Viscosity is incorporated indirectly via a stagnation condition at the leading edge (LE) and a Kutta condition at the trailing edge (TE).

(d) The total vorticity in the system is obtained as a sum of the bound and wake vorticity.

(e) The model is two dimensional, and is applied in a strip theory manner.

(f) The wake shed from each span-wise section is assumed to be confined to a cylindrical plane that is defined by the motion of the corresponding airfoil. Furthermore, each section is assumed to interact only with its shed wake. This assumption limits the applicability of the approach to the case of hover.

(g) Each airfoil is assumed to be rigid; however, the instantaneous values of airfoil chord, camber, and thickness can be used; thus, chordwise deformations are incorporated in a quasi-static manner.

(h) Each span-wise section of the wing is assumed to have three degrees of freedom: plunge $(h)$, lead-lag $(l)$, and pitch $(\alpha)$, as indicated in Figure 4(a). Furthermore, the degrees of freedom are defined with respect to the axis about which rigid body motion is prescribed.

\section{Model Formulation}

The wing is divided into several span-wise sections or airfoils; each airfoil is modeled as a continuous distribution of vorticity and the associated wakes are modeled using point vortices. A complex plane formulation, that transforms the airfoil into a circle using conformal mapping, is used to compute the flow field $;^{22}$ subsequently, an inverse transform is performed. The generalized Joukowski transform that is used to map each airfoil to a circle is given by

$$
\zeta=Z+\frac{(1-\varepsilon) R^{2}}{Z}+\frac{\varepsilon R^{3}}{2 Z^{2}}
$$

The vorticity and circulation are defined as positive when counterclockwise, as shown in Figure 4(b). The flow velocity $(q)$, vorticity $(\gamma)$, and circulation $(\Gamma)$, are transformed by

$$
q(\zeta)=\frac{q(Z)}{d \zeta / d Z} \quad ; \quad \gamma(\zeta)=\frac{\gamma(Z)}{d \zeta / d Z} \quad ; \quad \Gamma(\zeta)=\Gamma(Z)
$$

The total bound vorticity on the airfoil is obtained as a sum of two components: the quasi-steady vorticity that is obtained by ignoring the effect of the wake, and the wake-induced vorticity that is obtained by considering the effect of the entire wake:

$$
\gamma_{b}=\gamma_{q s}+\gamma_{w i}
$$

where, $\gamma_{q s}=\gamma_{f s}+\gamma_{u s}$. Following the derivation given in Ref. [22], $\gamma_{f s}$ and $\gamma_{u s}$ are given by

$$
\begin{aligned}
\gamma_{f s}(\theta, t)= & -2 U_{\infty}[\sin (\theta-\alpha)+\sin (\alpha)] \\
\gamma_{u s}(\theta, t)= & \frac{1}{R}\left[-A_{1} \cos \theta-\left(A_{2}+\frac{1}{2} A_{7}\right) \cos 2 \theta+A_{3} \sin \theta+\right. \\
& \left(A_{4}-\frac{1}{2} A_{5}+\frac{1}{2} A_{6}\right) \sin 2 \theta-A_{8} \sin \theta \cos 2 \theta+A_{9} \sin \theta \sin 2 \theta- \\
& \left.A_{10} \cos \theta \cos 2 \theta+A_{11} \cos \theta \sin 2 \theta+\frac{\left.\Gamma_{0}\right|_{u s}}{2 \pi}\right]
\end{aligned}
$$


where $A_{1}$ to $A_{11}$ are given in Ref. [22]. A Kutta condition at the TE is enforced so that $\gamma_{u s}(\theta=0, t)=0$. Therefore, $\left.\Gamma_{0}\right|_{u s}$ is obtained as:

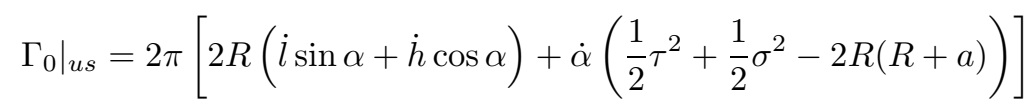

Integration of $\gamma_{f s}$ around the circle yields the circulation, $\left.\Gamma_{0}\right|_{f s}$, as:

$$
\left.\Gamma_{0}\right|_{f s}=-4 \pi R U_{\infty} \sin \alpha
$$

Therefore, the quasi-steady circulation, $\Gamma_{0}$, is obtained from Eqs. (12) and (13) as

$$
\begin{aligned}
\Gamma_{0} & =\left.\Gamma_{0}\right|_{f s}+\left.\Gamma_{0}\right|_{u s} \\
& =2 \pi\left[2 R\left(\left(i-U_{\infty}\right) \sin \alpha+\dot{h} \cos \alpha\right)+\dot{\alpha}\left(\frac{1}{2} \tau^{2}+\frac{1}{2} \sigma^{2}-2 R(R+a)\right)\right]
\end{aligned}
$$

The wake is modeled using point vortices that are shed from the TE and LE of the airfoil. The strength of the shed vortices is computed by enforcing a conventional Kutta condition at the TE and a stagnation condition at the LE based on the assumption that a streamline that separates at the LE re-attaches further downstream. ${ }^{22}$ The equations that are used to compute the strengths of the shed vortices are as follows:

At the TE:

$$
\Gamma_{0}=-\left[\oint_{w k} \mathcal{R}\left(\frac{Z_{w k}+R}{Z_{w k}-R}\right) \gamma_{w k} d Z_{w k}+\oint_{l e v} \mathcal{R}\left(\frac{Z_{l e v}+R}{Z_{l e v}-R}\right) \gamma_{l e v} d Z_{l e v}\right]
$$

and at the LE:

$$
\begin{aligned}
\frac{1}{R}\left[A_{1}-\left(A_{2}+\frac{1}{2} A_{7}\right)+A_{10}\right]-2 U_{\infty} \sin \alpha & =\frac{1}{2 \pi R}\left[\oint_{w k} \mathcal{R}\left(\frac{Z_{w k}-R}{Z_{w k}+R}\right) \gamma_{w k} d Z_{w k}\right. \\
& \left.+\oint_{l e v} \mathcal{R}\left(\frac{Z_{l e v}-R}{Z_{l e v}+R}\right) \gamma_{l e v} d Z_{l e v}\right]
\end{aligned}
$$

In Eqs. (15) and (16), note that the positions of the latest shed vortices, denoted by $Z_{w k}$ and $Z_{l e v}$, as well as the strengths, denoted by $\gamma_{w k}$ and $\gamma_{l e v}$, are unknown. The latest shed vortices are placed at specified distances from the TE and LE so that $\gamma_{w k}$ and $\gamma_{l e v}$ are the only remaining unknowns. ${ }^{23}$ Note that the positions and strengths of the vortices that were shed at previous time steps are known; therefore, Eqs. (15) and (16) are sufficient to determine the shed vorticity.

Solution of Eqs. (15) and (16), combined with the contributions of the previously shed vortices, yields the total vorticity in the wake. Then, the wake induced vorticity on the circle is computed as follows:

$$
\gamma_{w i}(\theta, t)=-\frac{\Gamma_{0}(t)}{2 \pi R}-\frac{1}{2 \pi R}\left[\oint_{w k} \mathcal{R}\left(\frac{Z_{w k}+R \mathrm{e}^{\imath \theta}}{Z_{w k}-R \mathrm{e}^{\imath \theta}}\right) \gamma_{w k} d Z_{w k}+\oint_{l e v} \mathcal{R}\left(\frac{Z_{l e v}+R \mathrm{e}^{\imath \theta}}{Z_{l e v}-R \mathrm{e}^{\theta \theta}}\right) \gamma_{l e v} d Z_{l e v}\right]
$$

It is assumed that the vorticity shed into the wake is convected using the Rott-Birkhoff equation. ${ }^{23,38}$

$$
q(\zeta)=\frac{\imath}{2 \pi} \oint \frac{d \Gamma^{\prime}}{\zeta-\zeta\left(\Gamma^{\prime}, t\right)}+\left(U_{E}-\imath V_{E}\right)
$$

where, $q(\zeta)$ denotes the complex velocity at a point with position vector $\zeta$ due to the action of a vortex in the system that has strength $d \Gamma^{\prime}$ and position vector $\zeta\left(\Gamma^{\prime}, t\right)$.

\section{Details of Implementation}

Implementation of the aerodynamic theory for rigid wings for the case of hover is described in Ref. [23]. Recall that each airfoil and its wake are assumed to be confined to a cylindrical plane; consequently, position vectors of the vortices in the airfoil-wake system are measured in the cylindrical plane. ${ }^{22}$ Implementation of the 
theory for flexible wings is similar; however, incorporating wing flexibility requires two changes: (1) For rigid wings, it is reasonable to assume that the cylindrical plane traced by each airfoil has a fixed radius, whereas for flexible wings, this radius is a function of the span-wise deformation of the wing; (2) For rigid wings and a symmetric upstroke and downstroke, the wing-wake interaction is predominantly two-dimensional in nature. For flexible wings, however, the paths traced by an airfoil in upstroke and downstroke may differ due to span-wise deformation and therefore, the wing-wake interaction is likely to be three-dimensional.

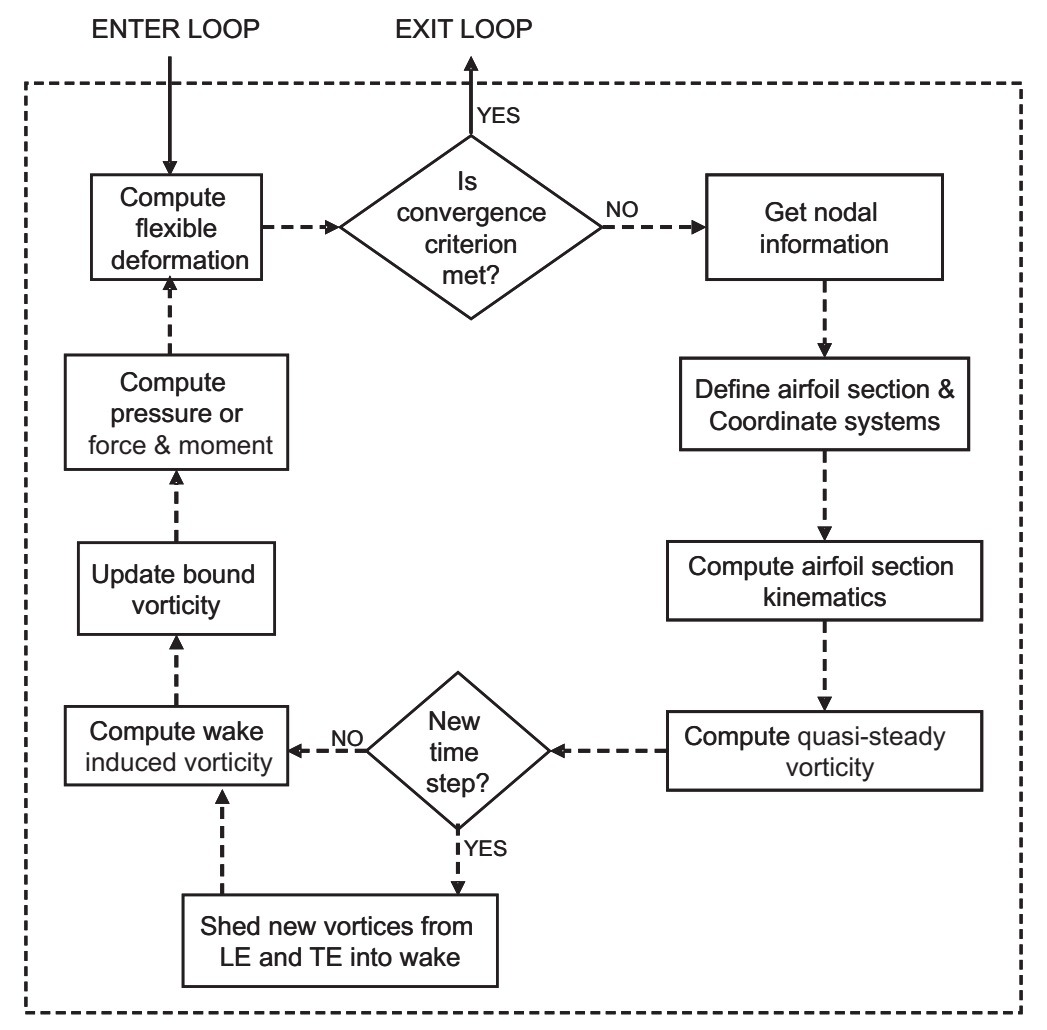

Figure 2. Schematic description of computation of aerodynamic loads.

For simplicity, it is assumed that the wing-wake interaction is two-dimensional. Distortion of the cylindrical plane due to span-wise deformation of the wing is accounted for in an approximate manner by incorporating an average time dependent radius that is a function of the paths traced by each airfoil at each time step. In Figure $3 R_{1}$ and $R_{2}$ denote the radii of the cylindrical plane at the start of the motion and at any subsequent time respectively. Then $R_{\text {Average }}=\left(R_{1}+R_{2}\right) / 2$.

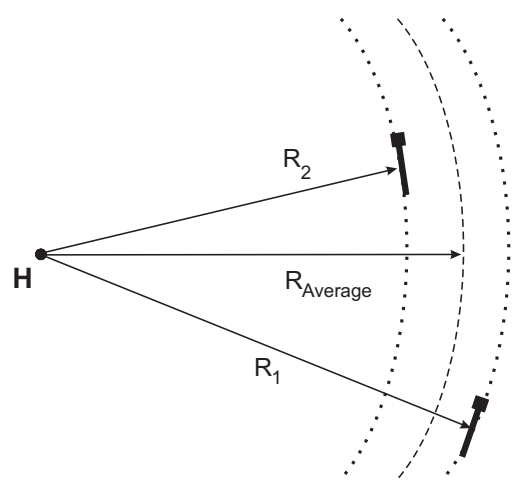

Figure 3. Average radius of the cylindrical plane for a wing that has span-wise deformation. 
Implementation of the aerodynamic model in the MARC user subroutine is shown in Figure 2 First, nodal positions and velocities, extracted from MARC, are used to define the airfoil coordinate systems, airfoil degrees of freedom, and compute the quasi-steady vorticity. Next, the most recent vortices are placed at specified distances from the LE and TE, and the strengths are computed using Eqs. (15) and (16). The vortices are shed into the wake only at the end of each time step and not for each step of the NewtonRaphson iteration. Finally, forces and moments, which are computed from the total vorticity in the system, are applied on the airfoil section. A brief description of the steps is provided next.

\section{$\underline{\text { Airfoil Geometric Parameters }}$}

Geometric parameters of the each airfoil that are required to compute aerodynamic loads include $R, \sigma$, and $\tau$ respectively. The current study is restricted to airfoils that have zero thickness; therefore, $\tau=0$. Computation of $R$ and $\sigma$ is described next.

The coordinate systems that are used in the analysis of each airfoil section are shown in Figure 4 and listed below:

(a) Airfoil coordinate system $\left(\mathcal{X}^{A}\right)$ : Origin at LE; $X^{A}$ along the chord; $Z^{A}$ normal to the chord.

(b) Global Inertial coordinate system $\left(\mathcal{X}^{G}\right)$ shown in Figure 5

(c) A non-rotating airfoil frame $\left(\mathcal{X}^{I}\right)$ : A frame that translates with $\mathcal{X}^{A}$, as shown in Figure 5 and is parallel to the $\hat{\zeta}$ coordinate system used in Ref. [22,23]. Position vectors in the airfoil-wake plane are measured with respect to the initial position of this frame.

(d) Airfoil coordinate system $\left(\mathcal{X}^{\zeta}\right)$ : Origin at the mid-chord; $X^{\zeta}$ along the zero-lift-line (ZLL); $Z^{\zeta}$ normal to ZLL as shown in Figure 4 This is equivalent to the $\zeta$ coordinate system used in Refs. [22, 23].

(e) Complex plane $(\mathcal{Z})$ : This is obtained via transformation of $\mathcal{X}^{\zeta}$ so that an airfoil of chord $c$ is transformed to a circle of radius $R$. Polar coordinates, $(R, \theta)$, are used to discretize the airfoil. Furthermore, the LE is located at $(-R, \pi)$ and TE is located at $(R, 0)$.

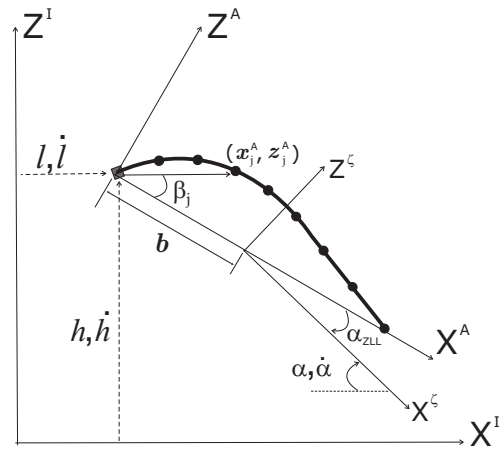

(a) Cartesian coordinate systems: axis about which rigid body motion is prescribed is shown at the leading edge

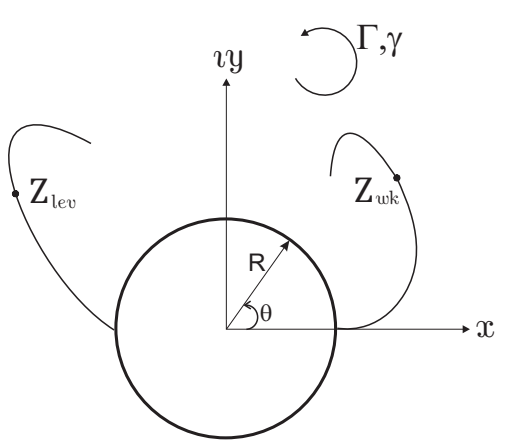

(b) Complex plane

Figure 4. Coordinate systems used in the aerodynamic analysis of the airfoil.

For each airfoil section, the zero-lift angle of attack is obtained from thin airfoil theory (pp. 68, Ref. [39]) as follows :

$$
\alpha_{z l l}=-\frac{2}{\pi} \sum_{j=2}^{n_{i}} \frac{z_{j}^{A}}{c} \frac{\Delta \beta_{j}}{\left(1+\cos \left(\beta_{j}\right)\right)}
$$

where $\beta_{j}=\tan ^{-1}\left(z_{j}^{A} / x_{j}^{A}\right) ; \beta_{1}=0$; and $\Delta \beta_{j}=\beta_{j}-\beta_{j-1}$. Then, $\mathcal{X}^{\zeta}$ is obtained from $\mathcal{X}^{A}$ by a rotation of $\alpha_{z l l}$ about $Y_{A}$ followed by a translation of the origin to the mid-chord. Note that placing the origin of the 


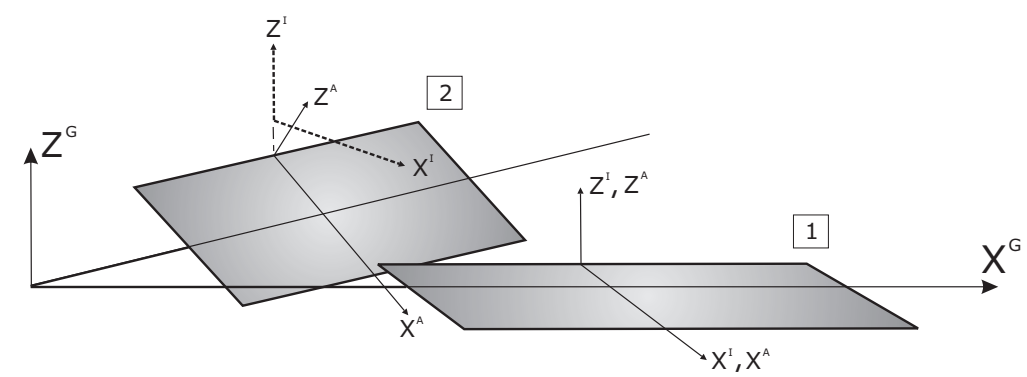

Figure 5. Coordinate systems on a wing that is undergoing prescribed motion.

circle at the mid-chord approximates the cambered airfoil as a circular arc. From Ref. [22],

$$
\begin{aligned}
\xi & =2 R \cos \theta+\sigma\left(\sin \theta-\frac{1}{2} \sin 2 \theta\right)-\tau\left(\cos \theta-\frac{1}{2} \cos 2 \theta\right) \\
\eta & =\tau\left(\sin \theta-\frac{1}{2} \sin 2 \theta\right)+\sigma\left(\cos \theta-\frac{1}{2} \cos 2 \theta\right)
\end{aligned}
$$

Substitute $\theta=0$ and $\theta=\pi$ to obtain coordinates of the TE and LE respectively.

$$
\left(\xi_{L E}, \eta_{L E}\right)=\left(-2 R,-\frac{3}{2} \sigma\right) \quad \text { and } \quad\left(\xi_{T E}, \eta_{T E}\right)=\left(2 R, \frac{1}{2} \sigma\right)
$$

Thus,

$$
R=\frac{\xi_{T E}-\xi_{L E}}{2} \quad \text { and } \quad \sigma=\frac{\eta_{T E}-\eta_{L E}}{2}
$$

\section{$\underline{\text { Vortex Placement }}$}

Position vectors of the vortices that are shed at the end of the first time step are given by: ${ }^{23}$

$$
\zeta_{\text {latest }}=\frac{1}{2} \bar{q} \Delta t
$$

Position vectors of the vortices shed at any subsequent time step during the analysis are given by: ${ }^{23}$

$$
\zeta_{\text {latest }}=\zeta_{\text {edge }}+\frac{1}{3}\left(\zeta_{\text {edge }}-\zeta_{\text {previous }}\right)
$$

\section{Vortex Wake Model}

Shed vortices are convected using Eq. (18). Note that Eq. (18) becomes singular as the separation between two vortices approaches zero. Therefore, numerical implementation of Eq. (18) requires desingularization of the vortex core. In this study, it is assumed that the shed vortices have a finite core radius. $^{23}$ Then, the discretized form of Eq. (18) modifies to the following equation:

$$
q\left(\zeta_{j}\right)=\frac{\imath}{2 \pi} \sum_{k=1}^{\infty} \frac{r_{j k} d \Gamma_{k}}{\sqrt{r_{c}^{4}+r_{j k}^{4}}} \mathbf{n}_{j k}+\left(U_{E}-\imath V_{E}\right)
$$

where $\infty$ indicates that the summation includes all the vortices in the airfoil-wake system. Furthermore, $\mathbf{n}_{j k}$ and $r_{j k}$ are obtained as follows:

$$
r_{j k}=\left\|\zeta_{j}\left(\Gamma_{j}, t\right)-\zeta_{k}\left(\Gamma_{k}, t\right)\right\|_{2} \quad \text { and } \quad \mathbf{n}_{j k}=\frac{\zeta_{j}\left(\Gamma_{j}, t\right)-\zeta_{k}\left(\Gamma_{k}, t\right)}{r_{j k}}, \quad r_{j k} \neq 0
$$

Reference [23] notes that the use of wake sub-iterations improves quality of the solution, especially when wake distortion due to wing-wake interaction is expected. Therefore, the Euler scheme ${ }^{23}$ in which the 
positions of the wake vortices are computed by performing several sub-iterations within each time step, is used.

$$
\zeta(t+\Delta t)=\zeta(t)+\sum_{j=1}^{n_{\text {sub }}} q_{j}(t) \frac{\Delta t}{n_{\text {sub }}}
$$

Vortex amalgamation algorithms are used to merge vortices in order to reduce the number of vortices in the system, thereby improving computational efficiency of the approach. ${ }^{23}$ In the current study, two vortices that have strengths $\Gamma_{i}$ and $\Gamma_{j}$ are merged if

$$
\Gamma_{i} \Gamma_{j}>0 \quad \text { and } \quad\left|\frac{\Gamma_{i} \Gamma_{j}}{\Gamma_{i}+\Gamma_{j}}\right| \delta_{i j}<\epsilon_{s e p}
$$

Computation of Aerodynamic Loads

The aerodynamic loads on each airfoil section are computed from the vorticity by using Kelvin's vortex impulse theorem. In this approach, the force and moment are obtained by computing the impulse and moment of impulse of the total vorticity in the airfoil-wake system. ${ }^{22}$ Following the derivation in Ref. [22], the force and moment in the non-rotating frame are computed as follows:

$$
\hat{F}=\frac{d \hat{I}}{d t} \quad \text { and } \quad \hat{M}=\frac{d \hat{I}_{m}}{d t}-\mathcal{I}\left(U_{0} \hat{I}\right)
$$

where $\mathcal{I}$ refers to the imaginary part and

$$
\hat{I}=\imath \rho_{\infty} \sum_{j=1}^{\infty} \Gamma_{j} \hat{\zeta}_{j} \quad ; \quad \hat{I}_{m}=\frac{\rho_{\infty}}{2} \sum_{j=1}^{\infty} \Gamma_{j}\left|\hat{\zeta}_{j}\right|^{2} \quad ; \quad U_{0}=\left(-U_{\infty}+i\right)+i \dot{h}
$$

\section{Formulation of the Aeroelastic Equations of Motion}

The equations of motion representing the aeroelastic response problem are obtained using an updated Lagrangian (UL) approach. ${ }^{40-42}$ In this approach, an approximate solution is obtained by referring all the quantities (stress, strain and displacements) of the deformed configuration to the equilibrium configuration obtained at the previous time step, and linearizing the resulting equations of motion (EOM). Implementation of the UL formulation in MARC, which is based on the description given in Refs. [40,42], is shown in Figure [6] and summarized below.

\section{A. The Updated Lagrangian Approach}

Starting from the equilibrium equations, the principle of virtual work yields the following integral form: ${ }^{40,42}$

$$
\int_{V^{t+\Delta t}} \sigma_{i j}^{t+\Delta t} \delta e_{i j}^{t+\Delta t} d V^{t+\Delta t}=\tilde{R}^{t+\Delta t}-F_{\text {inertial }}^{t+\Delta t}
$$

where

$$
\tilde{R}^{t+\Delta t}=\int_{A^{t+\Delta t}} T_{k}^{t+\Delta t} \delta u_{k}^{t+\Delta t} d A^{t+\Delta t}+\int_{V^{t+\Delta t}} \rho^{t+\Delta t} f_{k}^{t+\Delta t} \delta u_{k}^{t+\Delta t} d V^{t+\Delta t}
$$

and

$$
F_{\text {inertial }}^{t+\Delta t}=\int_{V^{t+\Delta t}} \rho^{t+\Delta t} \ddot{u}_{k}^{t+\Delta t} \delta u_{k}^{t+\Delta t} d V^{t+\Delta t}
$$

where, the right superscript on the quantities indicates that the deformed configuration, i.e. the configuration at time $t+\Delta t$, is the reference configuration.

The deformed configuration is unknown; thus, quantities that are referred to the deformed configuration, such as $\sigma_{i j}^{t+\Delta t}$ and $e_{i j}^{t+\Delta t}$, cannot be computed exactly. Therefore, the LHS of Eq. (31) is approximated by an 


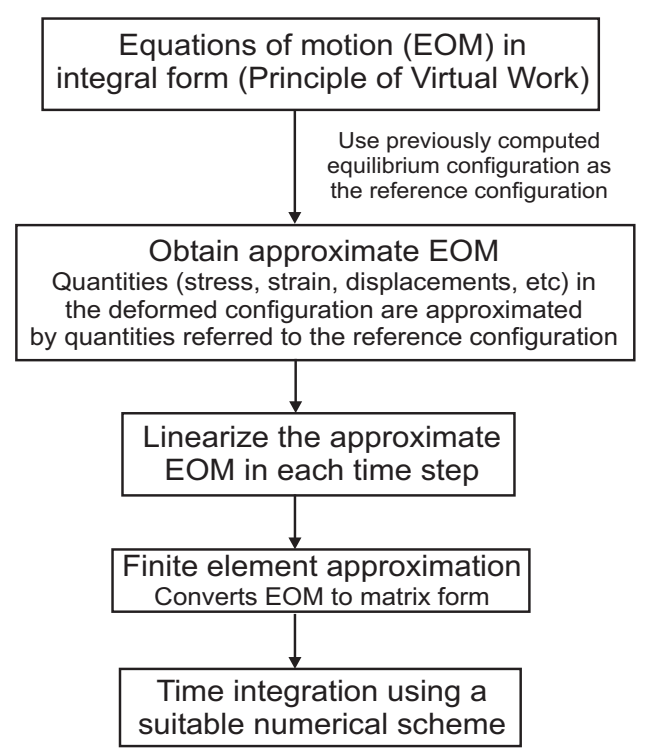

Figure 6. Schematic of the Updated Lagrangian Approach

integral that uses the configuration at time $t$ as the reference. In this approximation, $\sigma_{i j}^{t+\Delta t}$ is approximated by $S_{i j}^{t+\Delta t}$ and $e_{i j}^{t+\Delta t}$ is approximated by $\varepsilon_{i j}^{t+\Delta t}$. Therefore, Eq. (31) modifies to the following equation:

$$
\int_{V^{t}} S_{i j}^{t+\Delta t} \delta \varepsilon_{i j}^{t+\Delta t} d V^{t}=\tilde{R}^{t+\Delta t}-F_{\text {inertial }}^{t+\Delta t}
$$

Furthermore, the PK2 stress at time $t+\Delta t$ is obtained as a sum of the Cauchy stress in the reference configuration, $\sigma_{i j}^{t}$, and an incremental PK2 stress, $\Delta_{t} S_{i j}$ :

$$
S_{i j}^{t+\Delta t}=\sigma_{i j}^{t}+\Delta_{t} S_{i j}
$$

Similarly, the Green-Lagrange strain is given by

$$
\varepsilon_{i j}^{t+\Delta t}=\varepsilon_{i j}^{t}=e_{i j}^{t}+\eta_{i j}^{t}
$$

where $e_{i j}^{t}$ and $\eta_{i j}^{t}$ are obtained from the incremental displacement as

$$
\begin{aligned}
e_{i j}^{t} & =\frac{1}{2}\left(u_{i, j}^{t}+u_{j, i}^{t}\right) \\
\eta_{i j}^{t} & =\frac{1}{2} u_{k, i}^{t} u_{k, j}^{t}
\end{aligned}
$$

Furthermore, the constitutive relations are given by

$$
\Delta_{t} S_{i j}=C_{i j r s}^{t} \varepsilon_{r s}^{t}
$$

where $C_{i j r s}^{t}$ denotes the components of the tangent stiffness matrix. The final equation, obtained by substituting Eqs. (35), (36), and (38) into Eq. (34), is nonlinear in the incremental displacement $u_{i}$ and is given by

$$
\int_{V^{t}} C_{i j r s}^{t} \varepsilon_{r s}^{t} \delta \varepsilon_{i j}^{t} d V^{t}+\int_{V^{t}} \sigma_{i j}^{t} \delta \eta_{i j}^{t} d V^{t}=\tilde{R}^{t+\Delta t}-F_{\text {inertial }}^{t+\Delta t}-\int_{V^{t}} \sigma_{i j}^{t} \delta e_{i j}^{t} d V^{t}
$$

Equation (39) is linearized by assuming that $\delta \varepsilon_{i j}^{t}=\delta e_{i j}^{t}$, so that the constitutive equation becomes $\Delta_{t} S_{i j}=$ $C_{i j r s}^{t} e_{r s}^{t}$. Thus, the linearized integral equation that represents dynamics of the system is given by

$$
\int_{V^{t}} C_{i j r s}^{t} e_{r s}^{t} \delta e_{i j}^{t} d V^{t}+\int_{V^{t}} \sigma_{i j}^{t} \delta \eta_{i j}^{t} d V^{t}=\tilde{R}^{t+\Delta t}-F_{\text {inertial }}^{t+\Delta t}-\int_{V^{t}} \sigma_{i j}^{t} \delta e_{i j}^{t} d V^{t}
$$


The finite element approximation to the incremental displacements is given by

$$
\underline{u}(\underline{X}, t)=\underline{\mathbf{u}} \cdot \mathbf{N}^{t}
$$

Substituting Eqs. (41), (37), and (33), into Eq. (40), and simplifying the result yields the final form of the equation of motion as:

$$
\left(\mathbf{K}_{L}^{t}+\mathbf{K}_{N L}^{t}\right) \underline{\mathbf{u}}=\tilde{R}^{t+\Delta t}-\mathbf{M} \underline{\ddot{u}}^{t+\Delta t}-\underline{\mathbf{F}}^{t}
$$

Expressions for $\mathbf{K}_{L}^{t}, \mathbf{K}_{N L}^{t}, \underline{\mathbf{F}}^{t}$, and $\mathbf{M}$ are given below:

$$
\begin{aligned}
\mathbf{K}_{L}^{t} & =\int_{V^{t}}\left(\mathbf{B}_{L}^{t}\right)^{T} \mathbf{C}^{t} \mathbf{B}_{L}^{t} d V^{t} \\
\mathbf{K}_{N L}^{t} & =\int_{V^{t}}\left(\mathbf{B}_{N L}^{t}\right)^{T} \sigma^{t} \mathbf{B}_{N L}^{t} d V^{t} \\
\mathbf{F}^{t} & =\int_{V^{t}}\left(\mathbf{B}_{L}^{t}\right)^{T} \sigma^{t} d V^{t} \\
\mathbf{M} & =\int_{V^{t}}\left(\mathbf{N}^{t}\right)^{T} \rho^{t} \mathbf{N}^{t} d V^{t}
\end{aligned}
$$

where $\mathbf{B}_{L}^{t}$ and $\mathbf{B}_{N L}^{t}$ are transformation matrices that are obtained from the shape function matrices. The derivation of $\mathbf{B}_{L}^{t}$ and $\mathbf{B}_{N L}^{t}$ is described in Refs. [41,42]. Finally, Eq. (42), that represents the finite element discretization of the equation of motion, is integrated forward in time using an appropriate numerical scheme.

In this approach, the true stress and strain in the deformed configuration at the beginning of each time step are approximated using incremental stress and strain measures in the reference configuration. Therefore, iterations are often required for each time step so that the approximate incremental quantities converge. The iterations are done using the Newton-Raphson method. ${ }^{40,41}$ Two implementations of the method are available in MARC: ${ }^{33}$ (1) the complete Newton-Raphson method, wherein the tangent stiffness matrix is computed for each iteration in a time step, and (2) the modified Newton-Raphson method in which the stiffness matrix is assembled based on the initial, i.e. $t=0$, configuration. The complete Newton-Raphson method was used in this study. Description of the algorithm is presented in Refs. [33,40,41].

\section{B. Numerical Integration}

The equations of motion obtained from the UL method are integrated using a single step Houbolt (SSH) scheme. The implementation of this scheme in MARC is based on the description given in Ref. [43]. The SSH scheme, which belongs to the class of Houbolt algorithms, ${ }^{40,43}$ is an implicit and second order accurate scheme that is unconditionally stable for linear systems. By design, the algorithm incorporates numerical damping and is asymptotically annihilating, which implies that the high frequency response of the system is eliminated in each time step.

\section{Implementation of the Aeroelastic Model}

Implementation of the aeroelastic model is depicted in Figure $\mathbf{7}$ At each time step, rigid body motion is prescribed in an incremental manner using either rotation vectors or displacements. The aerodynamic loads computed based on the wing motion are applied to the structure via the FORCEM user subroutine in MARC. This subroutine is called from the main program for each step of the Newton-Raphson iteration to ensure convergence of the structural displacements and applied loads for each time step. Thus, the Newton-Raphson algorithm ensures the convergence of the aerodynamic loads and the corresponding flexible deformation of the wing within each time step. Finally, the vortices shed into the wake are convected at the end of the time step.

\section{Results and Discussion}

Capabilities of the MARC code were examined in order to determine its suitability for modeling flapping wing aeroelastic problems. Features of the code that are validated include: (1) Implementation of large 


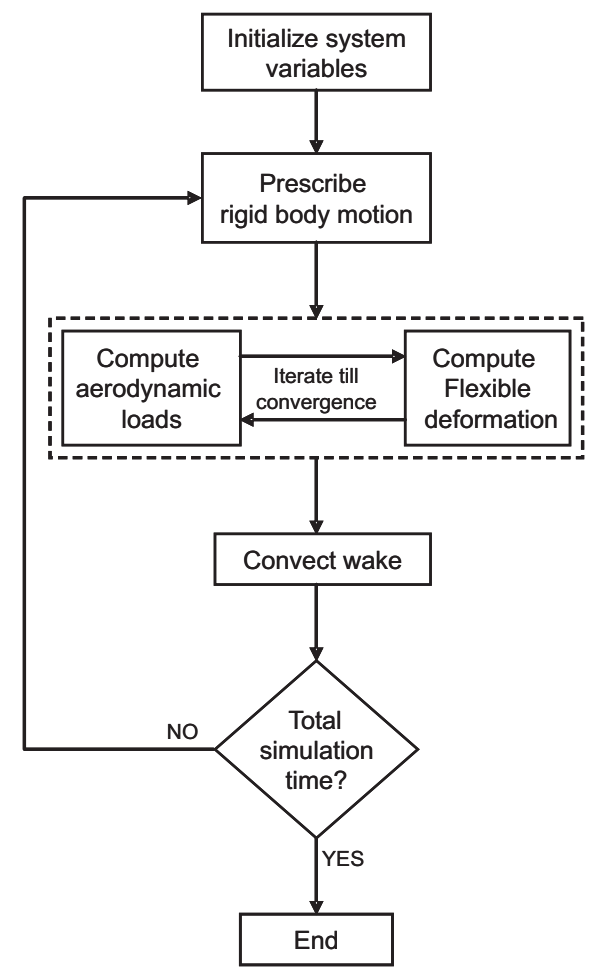

Figure 7. Schematic of the Aeroelastic Solution in MARC.

rigid body motions representative of wing kinematics (2) Application of pressure load on a structure that is undergoing prescribed rigid body motion. A preliminary validation of the approximate unsteady aerodynamic model has been presented in Ref. [23]. The model is tested further by considering two cases: (1) Airfoils undergoing prescribed plunge motion for a range of reduced frequencies and Strouhal numbers and (2) airfoils that have a fixed angle of attack in steady flow. Finally, the effect of flexibility on force generation is explored by considering flexible wings that are undergoing prescribed rigid body motion.

\section{A. Validation Studies}

\section{Wing Kinematics}

Implementation of large amplitude, time dependent rotations in MARC was validated by imposing two and three-dimensional rotations on a rigid plate that has dimensions $L=1 \mathrm{~m}$ and $b=0.25 \mathrm{~m}$. For the sake of conciseness, the results for the two-dimensional rotations are summarized. The rigid plate was modeled using a multi-point constraint (type RBE2) ${ }^{37}$ that allows several "slave" nodes to be rigidly linked to a single "master" node; subsequently, rotations were prescribed at the master node. In the plate configuration shown in Figure 8 the hinge point $\mathrm{H}$ was chosen as the master node and all other nodes were selected as slave nodes.

Implementation of two dimensional rotations was validated by imposing large amplitude rotations at the hinge $\mathrm{H}$. The $\mathrm{X}, \mathrm{Y}$, and $\mathrm{Z}$ displacements at a point $\mathrm{P}$ that has coordinates $(1,-0.25,0)$ in the plate-fixed frame were obtained from MARC and also independently calculated by implementing the equations in MATLAB. There was excellent agreement between the results, indicating that the rotation vector representation may be used to implement two dimensional rotations in MARC.

Next, a three dimensional rotation, described by the Euler angles given in Refs. [31,32], was provided as 


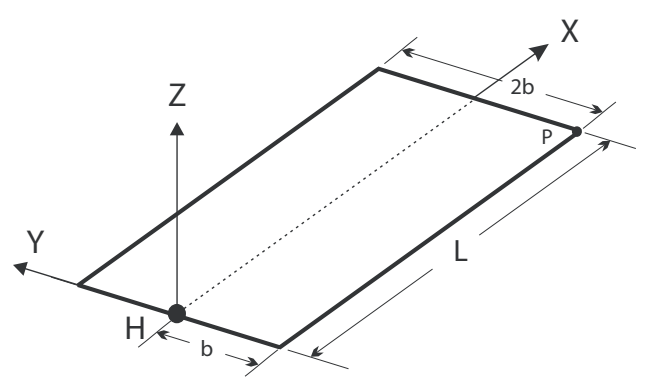

Figure 8. Rectangular plate

input to the rigid plate.

$$
\begin{aligned}
& \alpha=53^{\circ} \\
& \beta=-3^{o}-43^{\circ} \cos (2 \pi f t) \\
& \phi=8^{o}-77^{\circ} \cos \left(2 \pi f t-49^{\circ}\right)-3^{o} \cos \left(2 \pi f t+67^{\circ}\right)-8^{o} \cos \left(2 \pi f t+29^{\circ}\right)
\end{aligned}
$$

The Euler angles given in Eq. (47) represent a large rotation at $t=0$. In Ref. [31], this initial static rotation is used because the flapping motion was prescribed with respect to a body-fixed frame that did not initially coincide with the wing-fixed frame. For the case considered here, there is no initial offset between the body-fixed and plate-fixed frames; thus, the initial static rotation is eliminated by introducing Euler angles that correspond to the rotation at $t=0: \alpha_{0}=53^{\circ}, \beta_{0}=-46^{\circ}$, and $\phi_{0}=-50.7^{\circ}$.

The rotation matrix corresponding to the initial rotation, denoted by $\mathbf{R}_{0}$, is given by

$$
\mathbf{R}_{0}=\mathbf{R}_{\left(\alpha_{0}, \beta_{0}, \phi_{0}\right)}
$$

where $\mathbf{R}_{\left(\alpha_{0}, \beta_{0}, \phi_{0}\right)}$ is obtained using Eq. (6). The modified rotation matrix, denoted by $\mathbf{R}_{1}$, that describes the flapping motion without the initial rotation is given by

$$
\mathbf{R}_{1}=\mathbf{R}_{0}^{T} \mathbf{R}_{(\alpha, \beta, \phi)}
$$

The modified rotation tensor $\mathbf{R}_{1}$ is used to compute the rotation vector for a flapping frequency of $f=20$ $\mathrm{Hz}$ with a rotation increment of $10^{-4}$ radians. Figure 9 shows a comparison of displacements of the point $\mathrm{P}$ obtained from MARC with those computed using a MATLAB program. It is evident that using this implementation of rotations in MARC yields substantial errors. Decreasing the rotation increment to $10^{-6}$ radians did not produce improvement. This result implies that wing kinematics, which are typically threedimensional rotations, cannot be implemented as a time dependent rotation vector in MARC. Therefore, an alternate implementation of wing kinematics is considered next.

As an alternative to the time dependent rotation vector considered above, wing kinematics were represented as displacements. In this approach, displacements computed using the rotation tensor obtained in Eq. (49) were prescribed at the following points: the hinge $\mathrm{H}$, points $\mathrm{P}$ and $\mathrm{Q}$ that have coordinates (1.0,$0.25,0)$ and $(1.0,0.25,0)$ respectively in the plate-fixed frame. A comparison of the displacements of point $\mathrm{P}$ obtained from MARC to those computed using a MATLAB program is shown in Figure 9 There is excellent agreement, implying that wing kinematics may be implemented in MARC as displacements.

\section{Validation of the Fluid-Structure Interface}

Figure 10 shows a comparison of rotational velocity and acceleration of a rigid plate that is undergoing prescribed two-dimensional rotation. Note that rotational quantities are compared; therefore, dimensions of the plate are irrelevant. Quantities extracted from MARC using the NODVAR subroutine are compared to those calculated using a simple MATLAB program. The rotation is input using the following vector:

$$
\underline{\Psi}=\theta(t)\left\{\begin{array}{l}
0 \\
1 \\
0
\end{array}\right\}
$$



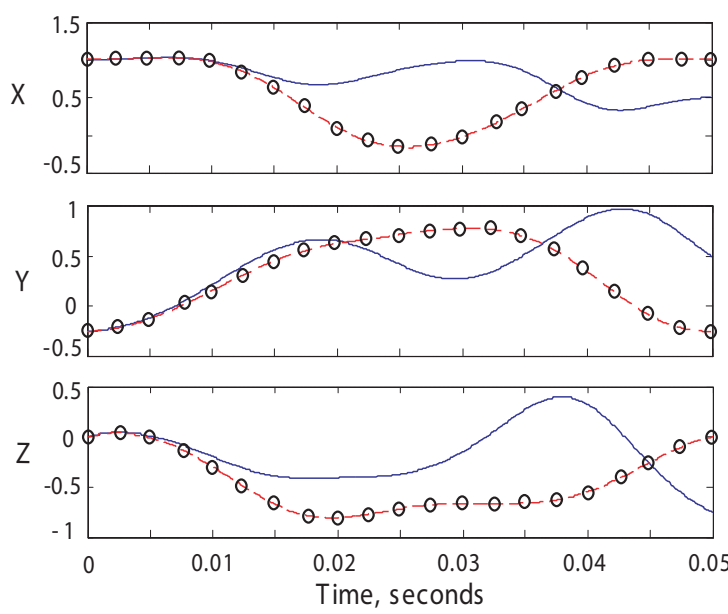

Figure 9. Implementation of rigid body rotations in MARC: solid line - kinematics imposed as rotations, circles - MATLAB implementation, dashed line - kinematics imposed as displacements.

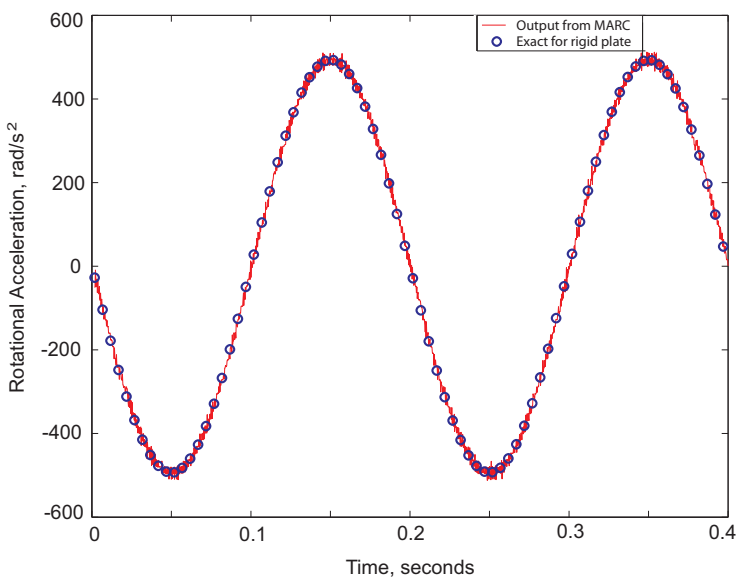

(a) Rotational Velocity

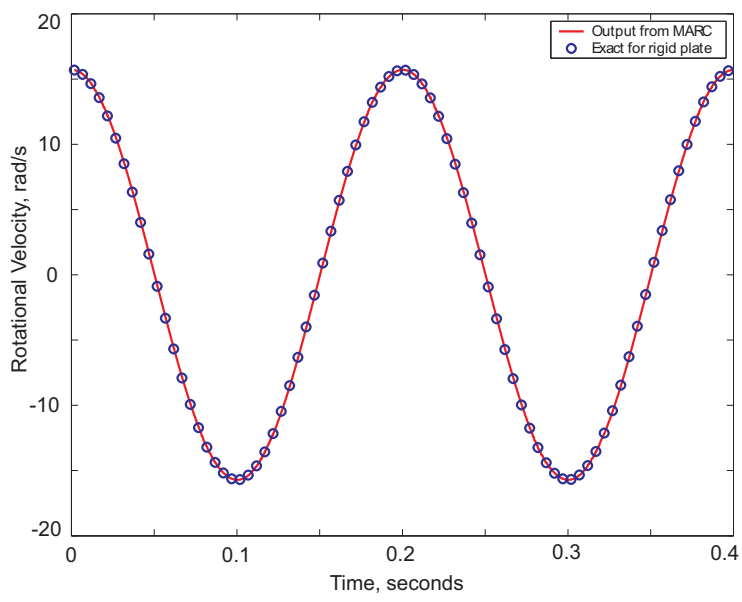

(b) Rotational Acceleration

Figure 10. Rotational velocity and acceleration: solid line - MARC, circles - MATLAB implementation

where $\theta(t)=0.5 \sin (2 \pi f t)$ radians, and $f=5 \mathrm{~Hz}$. Results show that the NODVAR subroutine may be reliably used to extract nodal quantities from MARC.

Next, uniform pressure load was applied on a flexible plate that is undergoing prescribed flapping motion. A plate with $L=0.1 \mathrm{~m}, b=0.0125 \mathrm{~m}$, and thickness $=0.001 \mathrm{~m}$, with $E=2.1 \times 10^{10} \mathrm{~N} / \mathrm{m}^{2}, \nu=0.3$, and $\rho=50 \mathrm{~kg} / \mathrm{m}^{3}$, was used. The low value of density was used so as to reduce the effect of inertial loads due to flapping motion. Thus, the edge displacements obtained for a flapping plate with external load are comparable to those obtained for a static plate that is cantilevered along the flapping edge with the same applied load. Two cases of uniform pressure loads, $\Delta p_{1}=500 \mathrm{~N} / \mathrm{m}^{2}$ and $\Delta p_{2}=10,000 \mathrm{~N} / \mathrm{m}^{2}$ were considered. The first case is intended to produce small deflections while the second case produces large deflections, compared to the thickness of the plate. For the flapping plate, the pressure loads were applied with large amplitude two-dimensional rotations described by Eq. (50).

A time step of $\Delta t=2.54 \times 10^{-4}$ seconds was used. At each time step, the magnitude of the displacement at the mid-point of the free edge due to flexibility, denoted by $\delta_{A B}$ where $A$ and $B$ are shown in Figure 11] is computed, where $\delta_{A B}=\sqrt{\delta_{l}^{2}+\delta_{p}^{2}}$, and $\delta_{l}$ and $\delta_{p}$ are shown in Figure 11] The results obtained for the static and flapping plates are given in Table 1. These results indicate that pressure loads can successfully 


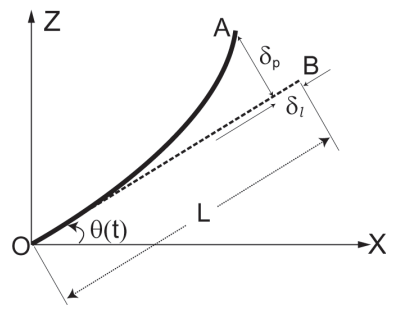

Figure 11. Side view of a flexible plate that is undergoing prescribed rigid body motion.

be imposed on a plate that is flapping with large amplitude rigid body motion.

\begin{tabular}{|c|c|c|}
\hline $\begin{array}{c}\text { Pressure } \\
N / m^{2}\end{array}$ & $\begin{array}{c}\text { Flapping case } \\
\times 10^{-3} \mathrm{~m}\end{array}$ & $\begin{array}{c}\text { Static case } \\
\times 10^{-3} \mathrm{~m}\end{array}$ \\
\hline 500 & 3.463 & 3.464 \\
10,000 & 62.6 & 63.7 \\
\hline
\end{tabular}

Table 1. Comparison of displacement $\delta_{A B}$ for static and flapping case.

3. Validation of the Aerodynamic Model
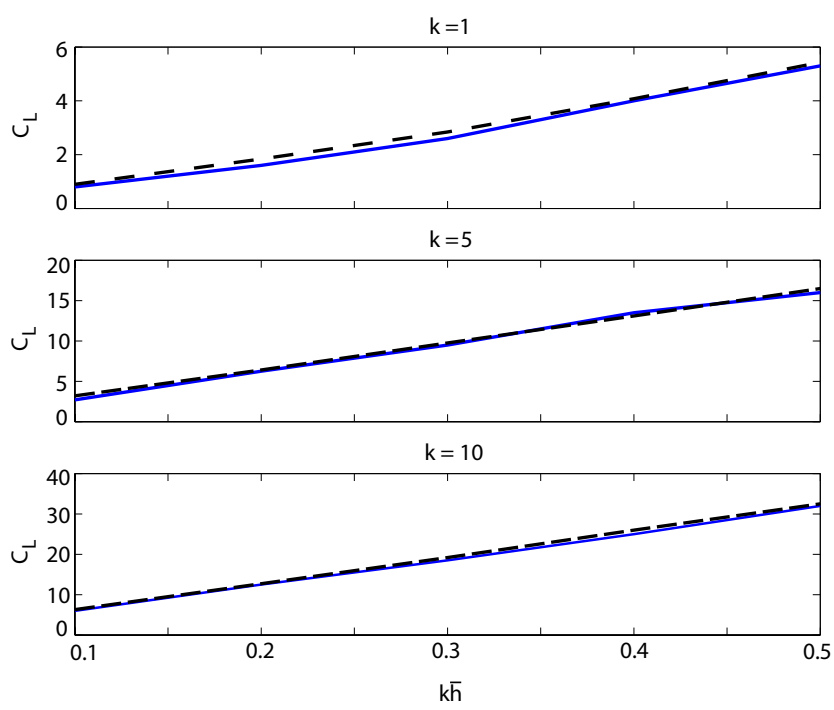

Figure 12. Comparison of lift coefficient for various reduced frequencies and non-dimensional plunge amplitudes; solid line - Ref. [20], dashed line - Current study.

First, a comparison of lift coefficients on plunging airfoils, computed by assuming that there was no leading edge separation, is shown in Figure 12. The results were obtained for $\operatorname{Re}=2 \times 10^{4}, k=1,5,10$, and a range of non-dimensional plunge amplitudes. Simulations using the approximate aerodynamic model were performed by using 50 points to discretize the airfoil, 3 wake sub-iterations, $r_{c}=0.04 c$, and $\epsilon_{\text {sep }}=1 \times 10^{-6}$. The results in Ref. [20] were obtained using an unsteady panel method that compared well with CFD computations based on NS equations. These results show excellent agreement between the forces predicted by the aerodynamic model and the unsteady panel method for the range of parameters considered.

Next, force coefficients, computed after a simulation time of $c / U_{\infty}$, for airfoils held at a fixed angle of attack are shown in Table 2 The results were obtained by assuming flow separation at the leading edge for 
an airfoil that has $c=0.1 \mathrm{~m}$ that is held at a 50 degree angle of attack to the free stream. Free stream velocities of $1.5 \mathrm{~m} / \mathrm{s}$ and $0.15 \mathrm{~m} / \mathrm{s}$, which correspond to Reynolds numbers of 1000 and 100 respectively, were considered. The CFD computations were performed using the CFD++ package, ${ }^{44}, 45$ wherein the flow field was computed using the incompressible, unsteady, Reynolds-averaged NS equations using a finite volume formulation, on a grid that has approximately 500,000 points and 200 points on the surface of the airfoil. Simulations using the approximate aerodynamic model were performed using the set of parameters used in the previous case. The results indicate that the aerodynamic model shows reasonable agreement with the CFD predictions.

\begin{tabular}{|c|c|c|c|c|}
\hline$U_{\infty}, \mathrm{m} / \mathrm{s}$ & Time step, seconds & & $C_{L}$ & $C_{D}$ \\
\hline \multirow{2}{*}{1.5} & $3.33 \times 10^{-4}$ & CFD & 2.08 & 2.48 \\
& $2 \times 10^{-3}$ & Approx Model & 2.5 & 2.8 \\
\hline 0.15 & $3.33 \times 10^{-4}$ & CFD & 1.82 & 2.16 \\
& $3.33 \times 10^{-4}$ & Approx Model & 2.1 & 2.5 \\
\hline
\end{tabular}

Table 2. Comparison of lift and drag coefficients: approximate model versus CFD.

\section{B. Preliminary Results for Fluid-Structure Interaction}

The effect of flexibility on the aerodynamic forces generated by wings undergoing prescribed rigid body motion is examined by performing "coupled" and "uncoupled" computations. A coupled computation implies that the wing deformation is computed by considering both aerodynamic and inertial loads; an uncoupled computation implies that wing deformation is computed using only inertial loads. These calculations are performed by assuming leading edge separation and for a zero free stream velocity, thereby simulating conditions of hover.

Two sets of rigid body motion are prescribed at the hinge, shown in Figure 13 (1) plunge motion described by Eq. (51) and (2) flap motion described by Eq. (52).

$$
\begin{aligned}
h(t) & =\bar{h} c(1-\cos (2 \pi f t)) \\
\beta(t) & =\beta_{0} \sin (2 \pi f t)
\end{aligned}
$$

A rectangular wing with a spar located at approximately one-third chord from the leading edge, shown in Figure 13, is used in this study. Geometric and material properties of the wing are listed below:

$\mathrm{L}=0.06 \mathrm{~m} ; \quad \mathrm{b}=0.0075 \mathrm{~m} ; \quad \mathrm{L}_{1}=0.01 \mathrm{~m} ; \quad \mathrm{w}_{1}=\mathrm{w}_{2}=1 \times 10^{-4} \mathrm{~m} ; \quad \mathrm{t}_{1}=\mathrm{t}_{2}=2 \times 10^{-5} \mathrm{~m} ;$ $\rho=1200 \mathrm{Kg} / \mathrm{m}^{3} ; \quad \nu=0.33 ; \quad$ Elastic modulus of the plate $=1 \mathrm{GPa} ; \quad$ Plate thickness $=1 \times 10^{-4} \mathrm{~m}$.

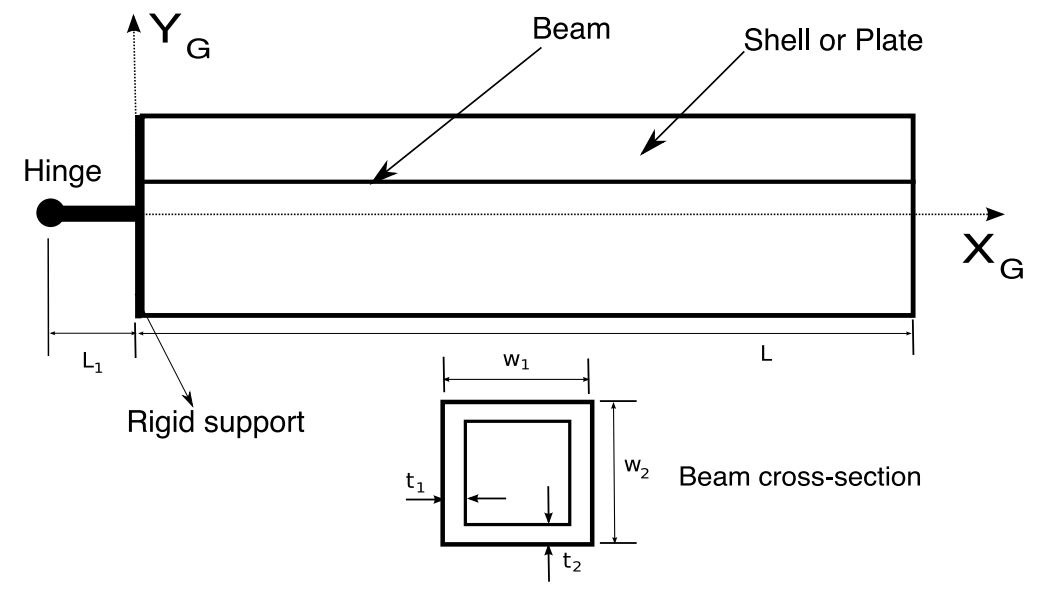

Figure 13. Wing that is used in this study. 
Note that width of the spar is equal to the thickness of the wing; therefore, the spar is embedded in the wing. The two configurations considered have different spar properties, and therefore different natural frequencies. These are labeled Config 1 and Config 2, respectively and their characteristics are listed in Table 3 The wing is modeled using four-noded shell elements, denoted by element type 75 in MARC. The

\begin{tabular}{|c|c|c|}
\hline Label & Elastic modulus of the spar & First natural frequency \\
\hline Config 1 & $0.1 \mathrm{GPa}$ & $27 \mathrm{~Hz}$ \\
Config 2 & $0.2 \mathrm{GPa}$ & $37 \mathrm{~Hz}$ \\
\hline
\end{tabular}

Table 3. Configurations used in this study

spar is modeled using two-noded beam elements (element type 78) that are based on Euler-Bernoulli theory and have global displacements and rotations as nodal degrees of freedom. The beam and shell elements are compatible so that beam-stiffened shell structures can be conveniently modeled.

The calculations are performed in air, $\rho_{\infty}=1.226 \mathrm{Kg} / \mathrm{m}^{3}$, and results were obtained for the following parameters:

Plunge: $\bar{h}=0.25, \mathrm{f}=10 \mathrm{~Hz}, 12 \mathrm{~Hz}, 14 \mathrm{~Hz}$

Flap: $\beta_{0}=0.087$ radians $\equiv 5$ degrees, $\mathrm{f}=8 \mathrm{~Hz}, 10 \mathrm{~Hz}, 12 \mathrm{~Hz}$

The simulations were performed using 100 points to discretize the airfoil, 15 wake sub-iterations, $r_{c}=0.04 c$, and $\epsilon_{\text {sep }}=1 \times 10^{-6}$. Furthermore, time steps were chosen so that each time period corresponding to the prescribed rigid body frequency was discretized using 100 to 150 points.

In several simulations, high frequency oscillations, such as shown in Figure 14, were observed in the aerodynamic forces generated by flapping as well as plunging wings. These numerical spikes were either minimal or totally absent in cases that had a non-zero free stream velocity and were most prominent in case that had a zero free stream velocity. The spikes, also reported in Ref. [23], are due to strong vortices that are released into the wake at each time step. These vortices result in large, non-physical, wake distortion thereby causing large fluctuations in aerodynamic loads. Furthermore, the fluctuations in the load signal increase significantly as the airfoil moves in the vicinity of such vortices. The spikes were reduced to a certain extent by reducing the size of the time step and increasing of the number of wake sub-iterations.

Low pass filters were used to eliminate the non-physical high frequency content in the time histories of the aerodynamic forces. Specifically, a Chebyshev function based filter that is available in the signal processing toolbox in MATLAB version 7.0.1 was used. Parameters which govern the smoothed curve were chosen so as to obtain a good fit with the original signal. A comparison of a typical load history and the corresponding smoothed response is shown in Figure 14.

Figures [15] and 16] show a comparison of lift, root bending moment, and non-dimensional tip displacement obtained from the coupled and uncoupled computations for the flexible configurations. Note that tip displacements were non-dimensionalized using the chord. Two cycles of oscillation corresponding to the rigid body frequency were considered. The difference in the results is relatively minor, indicating that the effect of aerodynamic loads on the wing is small compared to the effect of inertial loads.

Figures 17 and 18] show comparison of lift and tip displacement obtained for the rigid wing, Config 1, and Config 2. Results for the flexible configurations were obtained from a coupled computation. Figures 19] and 20 show a comparison of root bending moment for the flexible configurations. These results indicate that flexibility may have a favorable impact on lift generation; however, increasing wing flexibility has relatively minor impact on the forces and moments for the range of prescribed rigid body motions and flexibility considered.

\section{Conclusions}

The capability of the MARC code for analyzing the structural dynamic behavior of flapping wing micro air vehicles was found to be adequate. The coupling of a user defined unsteady aerodynamic program capable of representing leading edge vortices in addition to a conventional vortex wake shed from the trailing edge with MARC was implemented. Preliminary results for an insect-like wing configuration undergoing plunge and flap motions were generated. The results indicate that flexibility may have a favorable effect on lift generation; however the effect is fairly modest for the cases considered. 


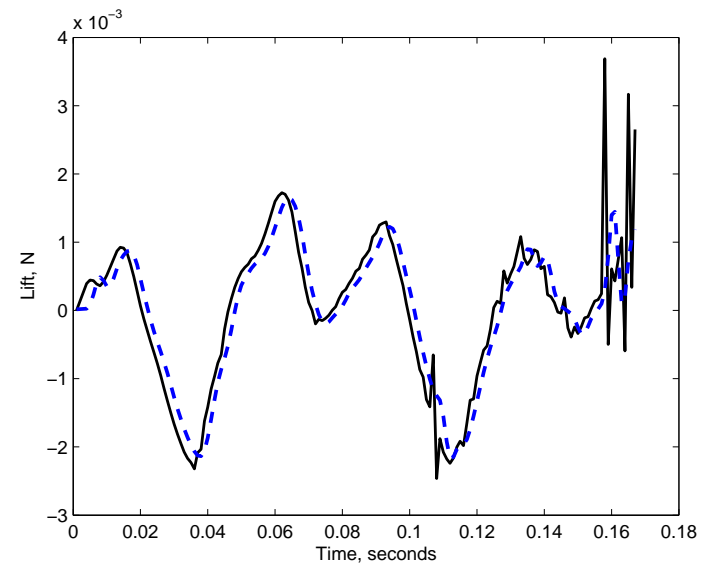

(a) $12 \mathrm{~Hz}$

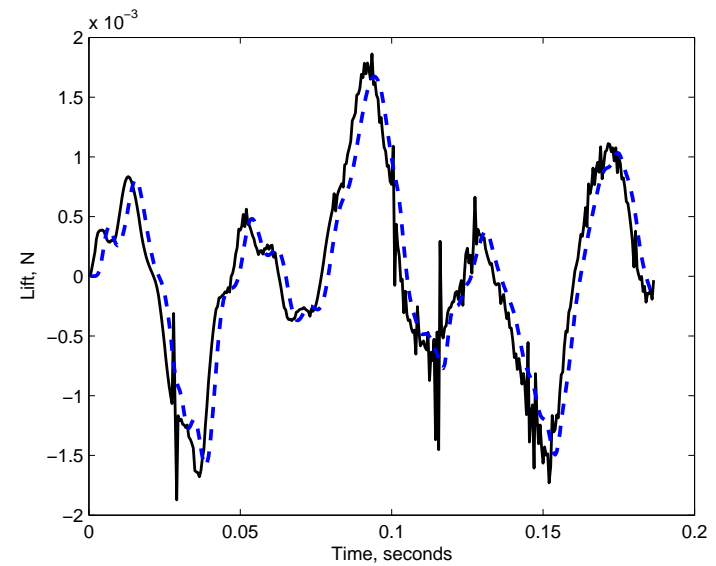

(b) $10 \mathrm{~Hz}$

Figure 14. Flap motion: Comparison of original signal and smoothed response; Lift obtained via uncoupled computation for Config 2; solid line - original signal, dashed line - smoothed response.

\section{Acknowledgments}

This research was funded by AFOSR Grant FA9550-06-1-0248 with Dr. Giugiutiu as program manager. Partial support from the AFOSR MURI project is also acknowledged. The authors thank Dr. L. Liu for generating the CFD results.

\section{References}

${ }^{1}$ Mueller, T. J., Fixed and Flapping Wing Aerodynamics for Micro Air Vehicle Applications, Vol. 195, Progress in Aeronautics and Astronautics, published by AIAA, 2001.

${ }^{2}$ Pines, D. J. and Bohorquez, F., "Challenges Facing Future Micro-Air-Vehicle Development," Journal of Aircraft, Vol. 43, No. 2, March - April 2006, pp. 290-305.

${ }^{3}$ Ellington, C. P., "The Aerodynamics of Hovering Insect Flight," Philosophical Transactions of the Royal Society of London B, Vol. 305, 1981, pp. 1 - 181.

${ }^{4}$ Platzer, M. E. and Jones, K., "Flapping Wing Aerodynamics - Progress and Challenges," $44^{\text {th }}$ AIAA Aerospace Sciences Meeting and Exhibit, Reno, Nevada, January 2006, AIAA Paper Number 2006-500.

${ }^{5}$ Shyy, W., Berg, M., and Ljungqvist, D., "Flapping and Flexible Wings for Biological and Micro Air Vehicles," Progress in Aerospace Sciences, Vol. 35, 1999, pp. 455 - 505.

${ }^{6}$ Sane, S. P., "The Aerodynamics of Insect Flight," The Journal of Experimental Biology, Vol. 206, 2003, pp. 4191 - 4208.

${ }^{7}$ Lian, Y., Shyy, W., Viieru, D., and Zhang, B., "Membrane Wing Aerodynamics for Micro Air Vehicles," Progress in Aerospace Sciences, Vol. 39, 2003, pp. $425-465$.

${ }^{8}$ Ansari, S. A., Zbikowski, R., and Knowles, K., "Aerodynamic Modelling of Insect-like Flapping Flight for Micro Air Vehicles," Progress in Aerospace Sciences, Vol. 42, 2006, pp. 129 - 172.

${ }^{9}$ Shyy, W., Lian, Y., Tang, J., Liu, H., Trizila, P., Stanford, B., Bernal, L., Cesnik, C., Friedmann, P., and Ifju, P., "Computational Aerodynamics of Low Reynolds Number Plunging, Pitching, ad Flexible Wings for MAV Applications," 48 ${ }^{t h}$ AIAA Aerospace Sciences Meeting and Exhibit, Reno, Nevada, January 2008, AIAA Paper No. 2008-523.

${ }^{10}$ Shyy, W., Lian, Y., Tang, J., Viieru, D., and Liu, H., Aerodynamics of Low Reynolds Number Flyers, Cambridge University Press, 2008.

${ }^{11}$ Bisplinghoff, R. L., Ashley, H., and Halfman, R. L., Aeroelasticity, Addison Wesley Co., 1955.

${ }^{12}$ Ellington, C. P., van den Berg, C., Willmott, A. P., and Thomas, A. L. R., "Leading-Edge Vortices in Insect Flight," Nature, Vol. 384, 1996, pp. $626-630$.

${ }^{13}$ Birch, J. M., Dickson, W. B., and Dickinson, M. H., "Force Production and Flow Structure of the Leading Edge Vortex on Flapping Wings at High and Low Reynolds number," The Journal of Experimental Biology, Vol. 207, 2004 , pp. 1063 - 1072.

${ }^{14}$ Shyy, W. and Liu, H., "Flapping Wings and Aerodynamic Lift: The Role of Leading-Edge Vortices," AIAA Journal, Vol. 45, No. 12, 2007, pp. $2817-2819$.

${ }^{15}$ Singh, B. and Chopra, I., "An Aeroelastic Analysis for the Design of Insect-Based Flapping Wings," $48^{t h}$ AIAA/ASME/ASCE/AHS/ASC Structures, Structural Dynamics, and Materials Conference, Honolulu, Hawaii, April 2007, AIAA Paper Number 2007-1757.

${ }^{16}$ Singh, B., Dynamics and Aeroelasticity of Hover Capable Flapping Wings: Experiments and Analysis, Ph.D. thesis, University of Maryland, College Park, 2006. 
${ }^{17}$ Azuma, A. and Okomoto, M., "Theoretical Study on Two-Dimensional Aerodynamic Characteristics of Unsteady Wings," Journal of Theoretical Biology, Vol. 40, May 2005, pp. $67-78$.

${ }^{18}$ Polhamus, E. C., "A Concept of the Vortex Lift of Sharp Edge Delta Wings Based on a Leading-Edge Suction Analogy," Nasa tn d-3767, 1966.

${ }^{19}$ Smith, M. J., "Simulating Moth Wing Aerodynamics," AIAA Journal, Vol. 34, No. 7, 1996, pp. 1348 - 1355.

${ }^{20}$ Young, J. and Lai, J. C. S., "Oscillation Frequency and Amplitude Effects on the Wake of a Plunging Airfoil," AIAA Journal, Vol. 42, No. 10, 2004, pp. $2042-2052$.

${ }^{21} \mathrm{Katz}$, J., "A Discrete Vortex Method for the Non-steady Separated Flow over an Airfoil," Journal of Fluid Mechanics, Vol. 102, 1981, pp. 315-328

${ }^{22}$ Ansari, S. A., Zbikowski, R., and Knowles, K., "Non-linear Unsteady Aerodynamic Model for Insect-like Flapping Wing in the Hover. Part 1: Methodology and Analysis," Proceedings of the I MECH E Part G Journal of Aerospace Engineering, Vol. 220, No. 2, 2006, pp. 61-83.

${ }^{23}$ Ansari, S. A., Zbikowski, R., and Knowles, K., "Non-linear Unsteady Aerodynamic Model for Insect-like Flapping Wing in the Hover. Part 1: Implementation and Validation," Proceedings of the I MECH E Part G Journal of Aerospace Engineering, Vol. 220, No. 2, 2006, pp. 169-186.

${ }^{24}$ Tang, J., Viieru, D., and Shyy, W., "Effects of Reynolds Number and Flapping Kinematics on Hovering Aerodynamics," $45^{\text {th }}$ AIAA Aerospace Sciences Meeting and Exhibit, Reno, Nevada, January 2007, AIAA Paper Number 2007-129.

${ }^{25}$ Tang, J., Viieru, D., and Shyy, W., "A Study of Aerodynamics of Low Reynolds Number for Flexible Airfoils," $37^{\text {th }}$ AIAA Fluid Dynamics Conference and Exhibit, Miami, FL, June 2007, AIAA Paper Number 2007-4212.

${ }^{26}$ Wootton, R. J., "Functional Morphology of Insect Wings," Annual Review of Entymology, Vol. 37, 1992, pp. 113 - 140.

${ }^{27}$ Wootton, R. J., Herbert, R. C., Young, P. G., and Evans, K. E., "Approaches to Modeling of Insect Wings," Philosophical Transactions of the Royal Society of London B, Vol. 358, 2003, pp. 1577 - 1587.

${ }^{28}$ Combes, S. A. and Daniel, T. L., "Flexural Stiffness in Insect Wings: I. Scaling and Influence of Wing Venation," The Journal of Experimental Biology, Vol. 206, 2003, pp. 2979 - 2987.

${ }^{29}$ Combes, S. A. and Daniel, T. L., "Into Thin Air: Contributions of Aerodynamic and Inertial-Elastic Forces to Wing Bending in the Hawkmoth Manduca Sexta," The Journal of Experimental Biology, Vol. 206, 2003, pp. 2999 - 3006.

${ }^{30}$ Daniel, T. L. and Combes, S. A., "Flexible Wings and Fins: Bending by Inertial or Fluid-Dynamic Forces," Integrative and Comparative Biology, Vol. 42, 2002, pp. 1044 - 1049.

${ }^{31}$ Barut, A., Das, M., and Madenci, E., "Nonlinear Deformations of Flapping Wings on a Micro Air Vehicle," $47^{t h}$ AIAA/ASME/ASCE/AHS/ASC Structures, Structural Dynamics, and Materials Conference, Newport, Rhode Island, May 2006, pp. 184-196, AIAA Paper Number 2006-1662.

${ }^{32}$ Azuma, A., Azuma, S., Watanabe, I., and Furuta, T., "Flight Mechanics of a Dragonfly," Journal of Experimental Biology, Vol. 116, 1985, pp. $79-107$.

${ }^{33}$ MSC. MARC, Volumes A - D, 2005.

${ }^{34}$ Bauchau, O. A. and Trainelli, L., "The Vectorial Parametrization of Rotation," Nonlinear Dynamics, Vol. 32, No. 1, 2003, pp. $71-92$.

${ }^{35}$ Kane, T. R., Likins, P. W., and Levinson, D. A., Spacecraft Dynamics, Mc-Graw Hill Publications, 1983.

${ }^{36}$ Baruh, H., Analytical Dynamics, Mc-Graw Hill Publications, 1999.

${ }^{37}$ MSC. NASTRAN, Reference Manual, 2004.

${ }^{38}$ Saffman, P. G., Vortex Dynamics, Cambridge University Press, 1992

${ }^{39}$ Abbott, I. H. and von Doenhoff, A. E., Theory of Wing Sections: Including a Summary of Airfoil Data, Dover Publications, 1959

${ }^{40}$ Bathe, K., Finite Element Procedures, Prentice-Hall Inc., 1996.

${ }^{41}$ Belytschko, T., Liu, W. K., and Moran, B., Nonlinear Finite Elements for Continua and Structures, Wiley \& Sons Ltd., 2001.

${ }^{42}$ Bathe, K., Ramm, E., and Wilson, E. L., "Finite Element Formulations for Large Deformation Dynamic Analysis," International Journal for Numerical Methods in Engineering, Vol. 9, 1975, pp. 353 - 386.

${ }^{43}$ Chung, J. and Hulbert, G. M., "A Family of Single-Step Houbolt Time Integration Algorithms for Structural Dynamics," Computational Methods in Applied Mechanics and Engineering, Vol. 118, 1994, pp. 1 - 11.

${ }^{44}$ Peroomian, O., Chakravarthy, S., Palaniswamy, S., and Goldberg, U., "Convergence Acceleration for Unified-Grid Formulation Using Preconditioned Implicit Relaxation," Reno, NV, January 1998, AIAA Paper 98-0116.

${ }^{45}$ Peroomian, O., Chakravarthy, S., and Goldberg, U., "A "Grid-Transparent" Methodology for CFD," Reno, NV, January 1997, AIAA Paper 97-0724. 


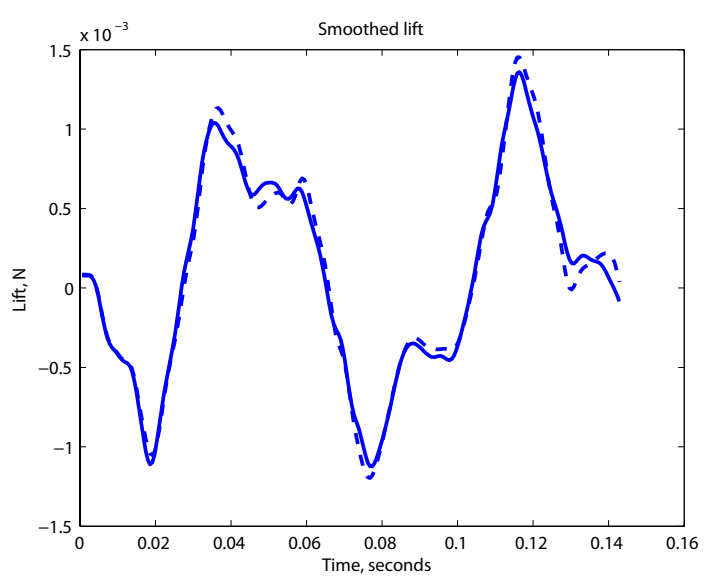

(a) Config 1: Lift

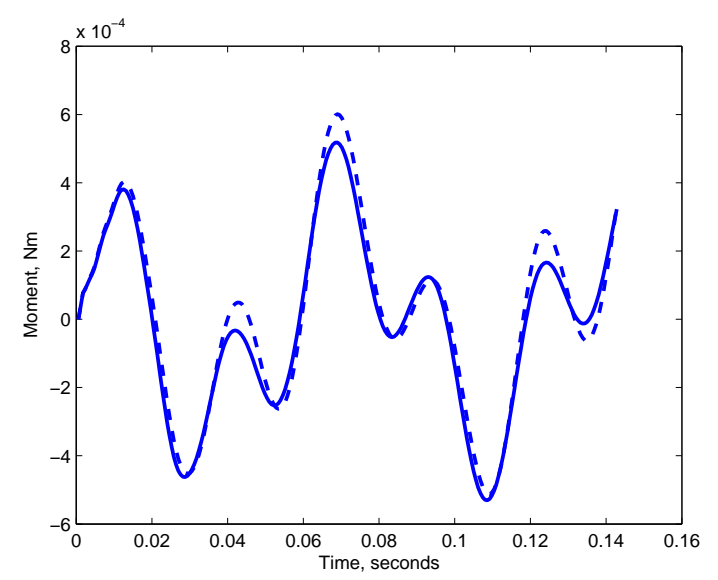

(c) Config 1: Root Bending Moment

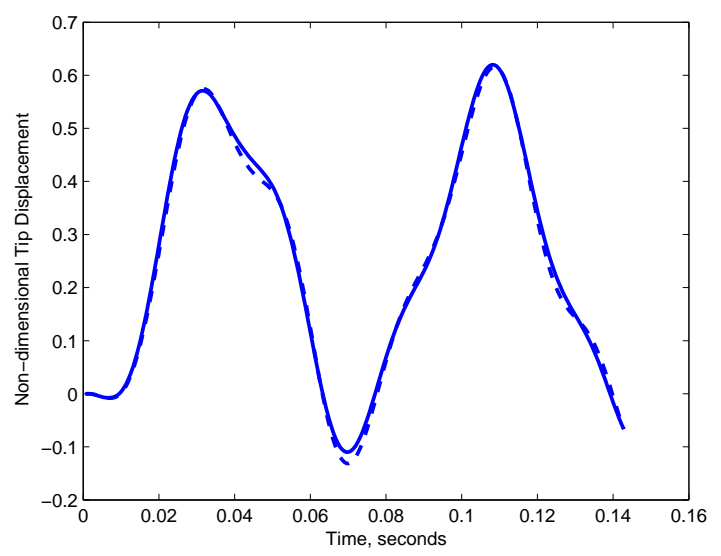

(e) Config 1: Tip displacement

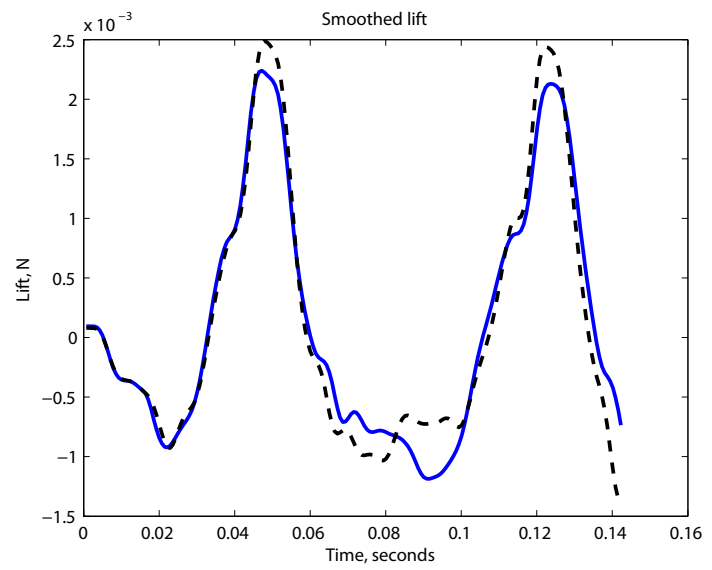

(b) Config 2: Lift

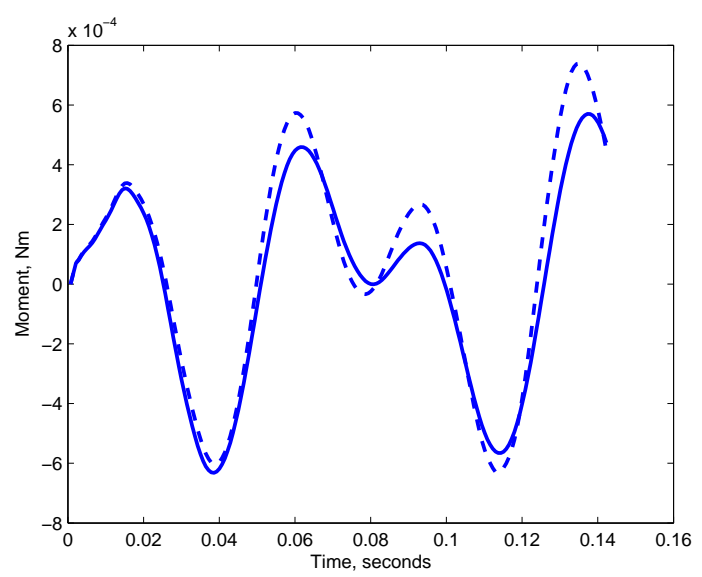

(d) Config 2: Root Bending Moment

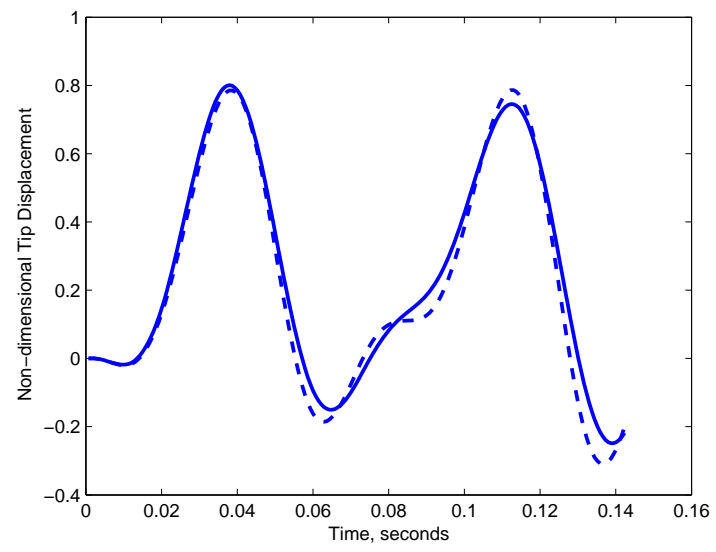

(f) Config 2: Tip Displacement

Figure 15. Plunge motion: Coupled and uncoupled response, $14 \mathrm{~Hz}$; solid line - uncoupled, dashed line coupled. 


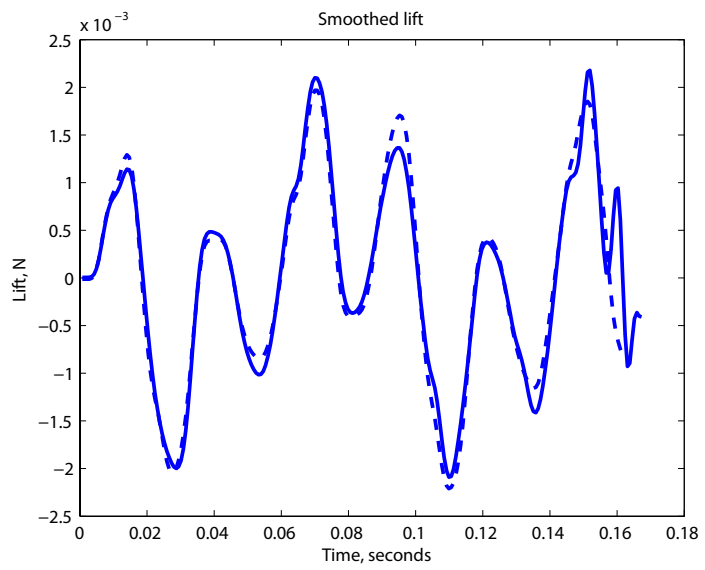

(a) Config 1: Lift

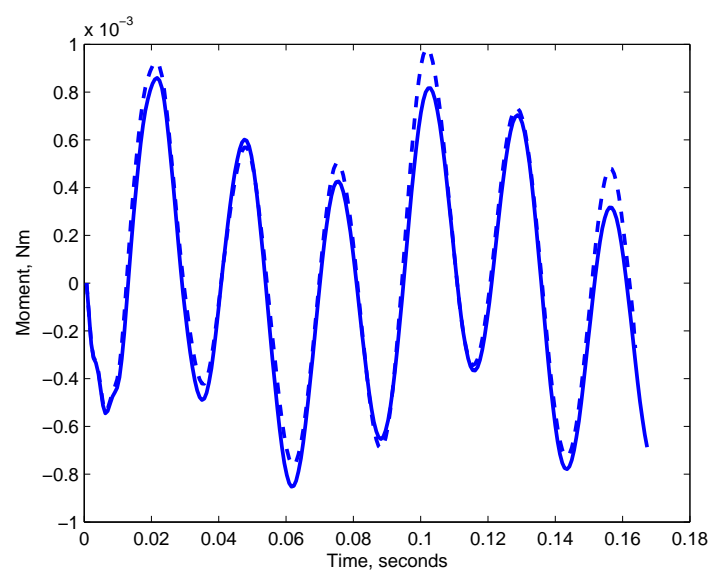

(c) Config 1: Root Bending Moment

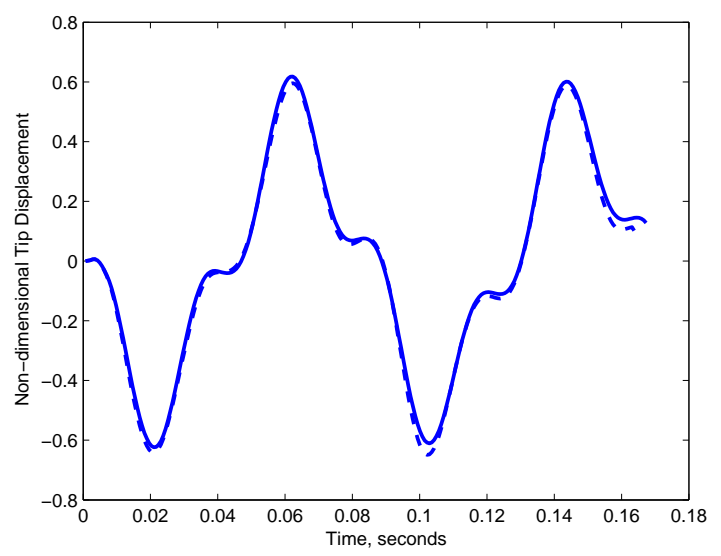

(e) Config 1: Tip displacement

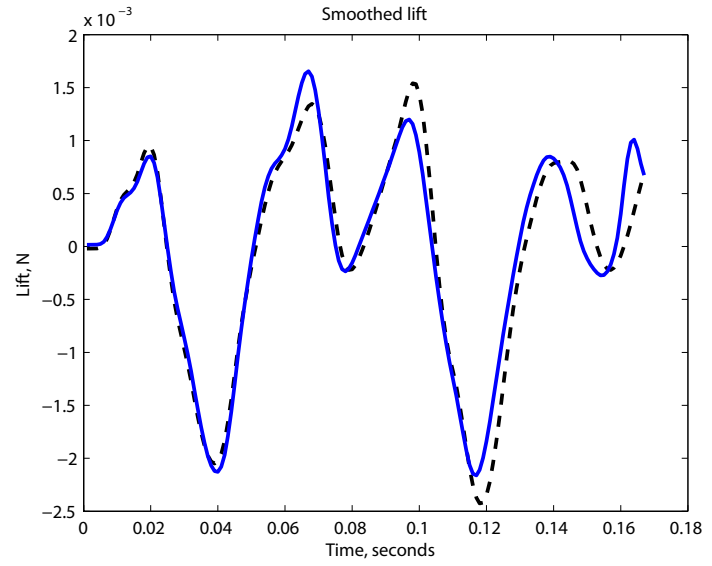

(b) Config 2: Lift

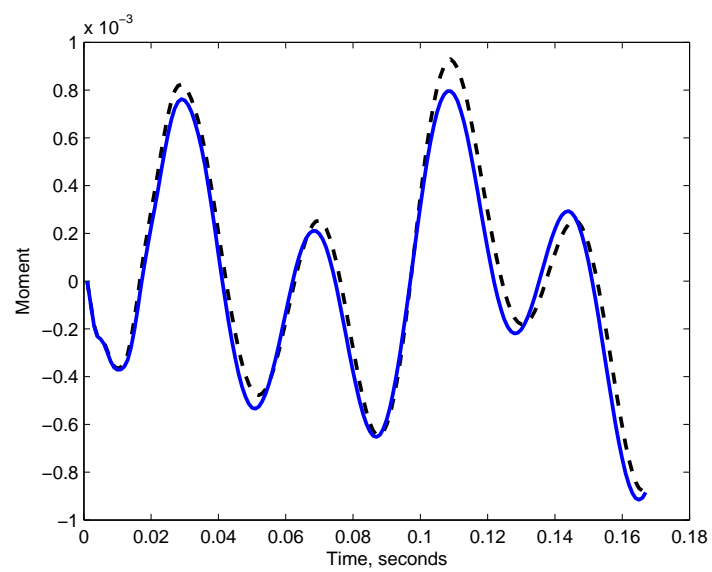

(d) Config 2: Root Bending Moment

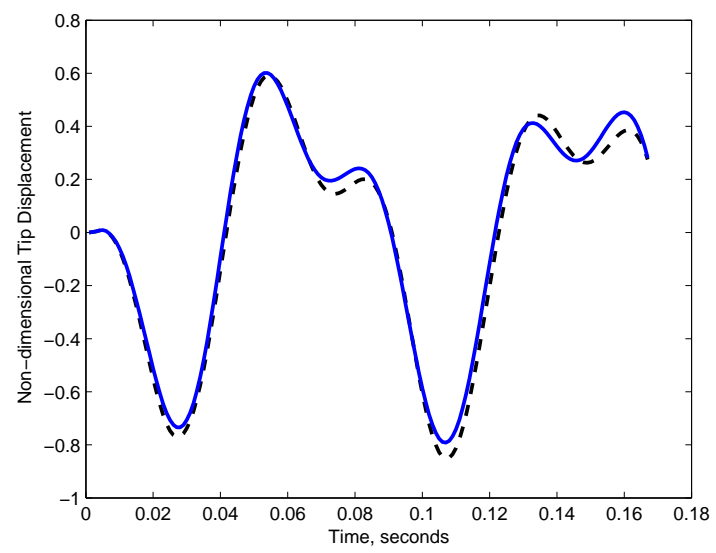

(f) Config 2: Tip Displacement

Figure 16. Flap motion: Coupled and uncoupled response, $12 \mathrm{~Hz}$; solid line - uncoupled, dashed line coupled. 


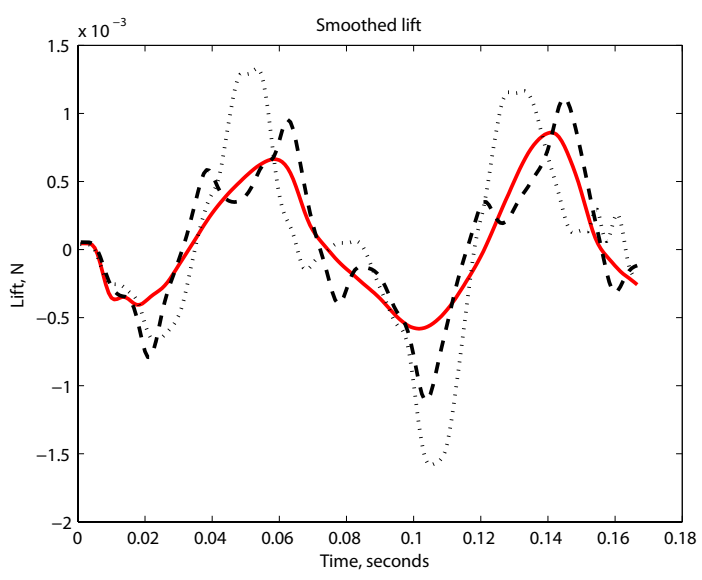

(a) $14 \mathrm{~Hz}$ : Lift

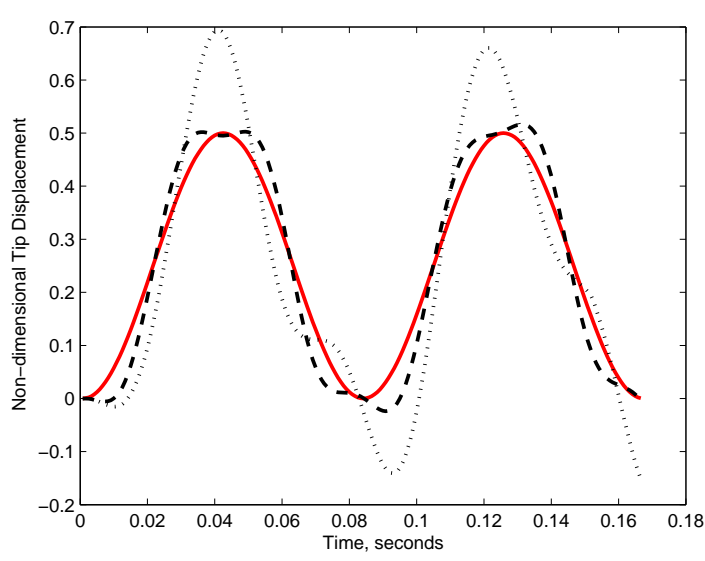

(c) $14 \mathrm{Hz:}$ Tip displacement

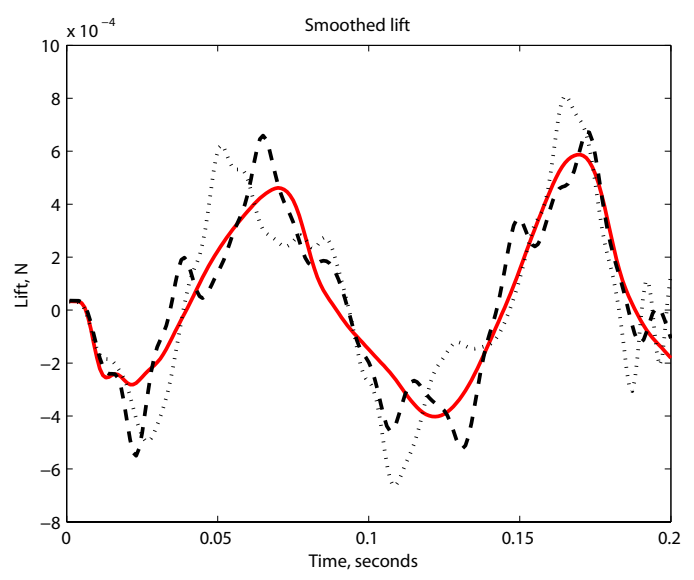

(b) $12 \mathrm{Hz:}$ Lift

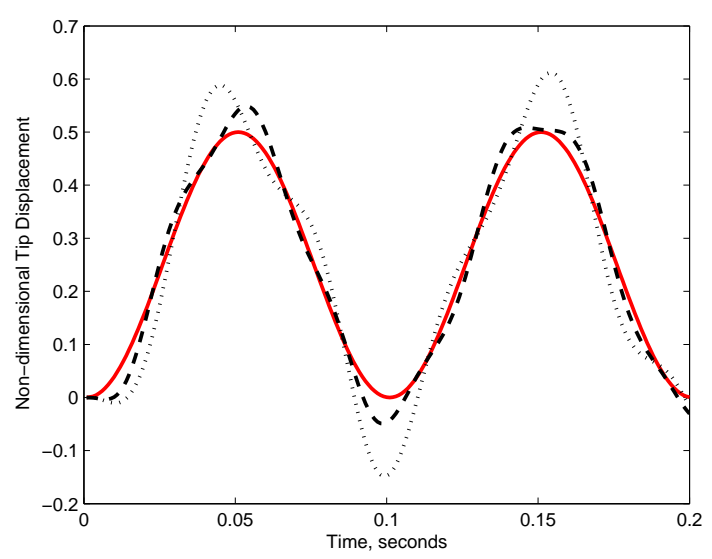

(d) 12 Hz: Tip Displacement

Figure 17. Plunge motion: Comparison of Rigid, Config 1 and Config 2 ; solid line - rigid, dashed line - Config 1, dotted line - Config 2 


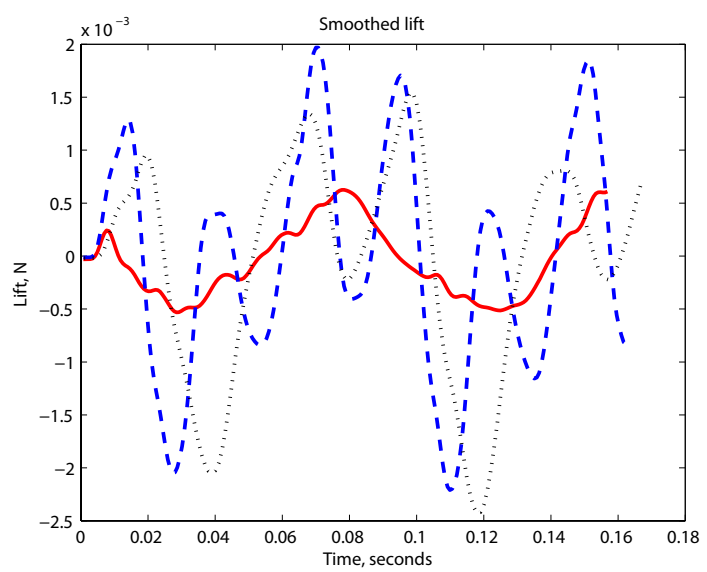

(a) $12 \mathrm{~Hz}:$ Lift

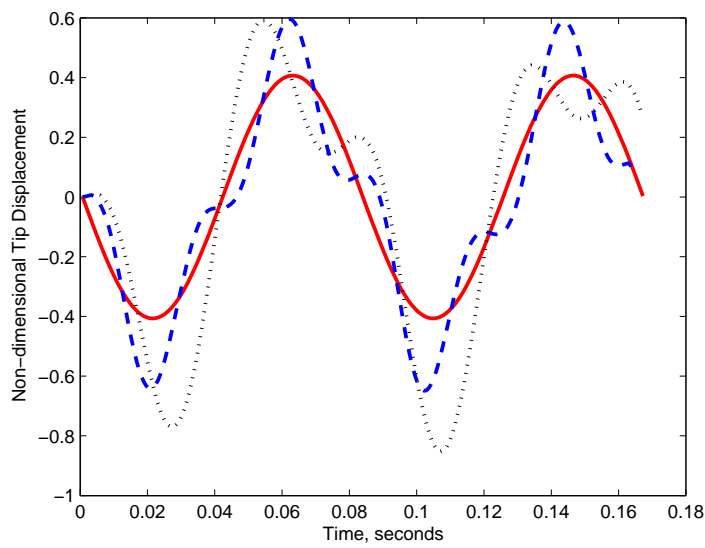

(c) 12 Hz: Tip displacement

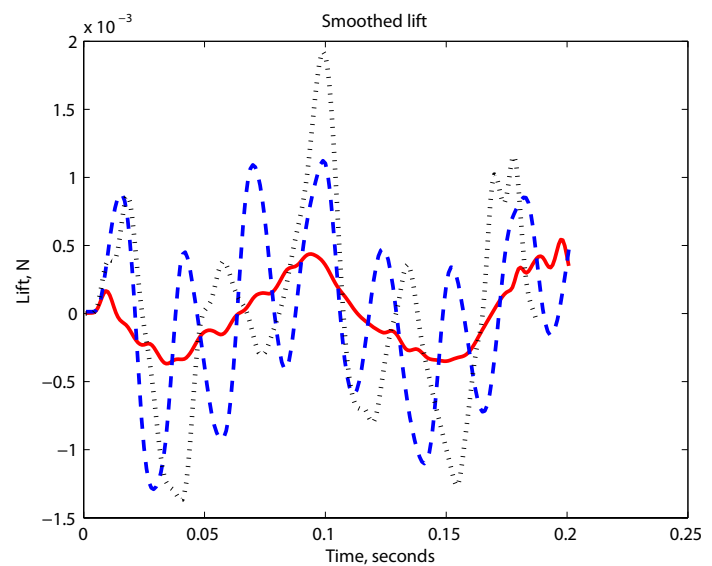

(b) $10 \mathrm{~Hz}$ : Lift

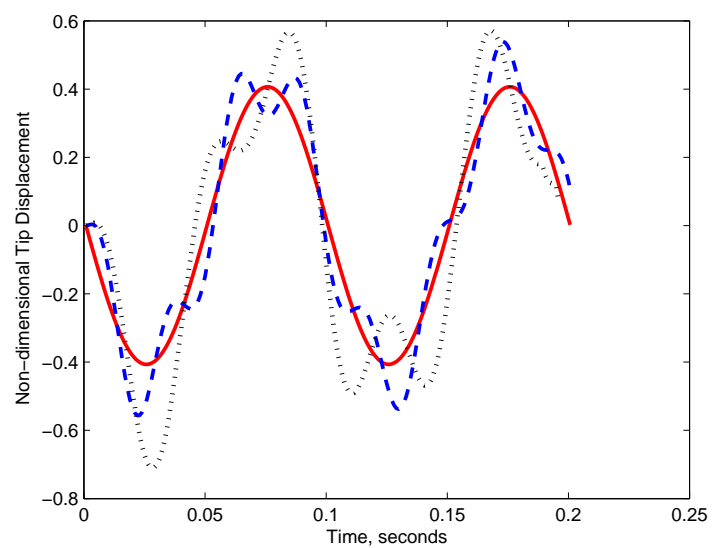

(d) $10 \mathrm{Hz:}$ Tip Displacement

Figure 18. Flap motion: Comparison of Rigid, Config 1 and Config 2 ; solid line - rigid, dashed line - Config 1 , dotted line - Config 2

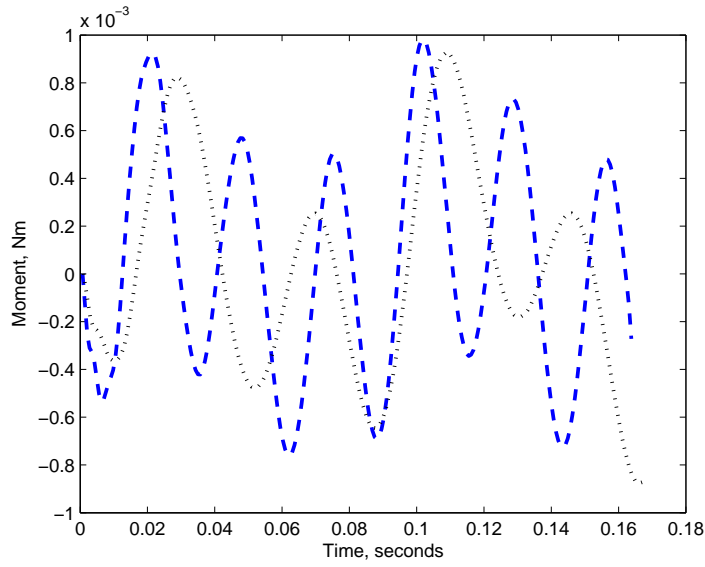

(a) $12 \mathrm{~Hz}$

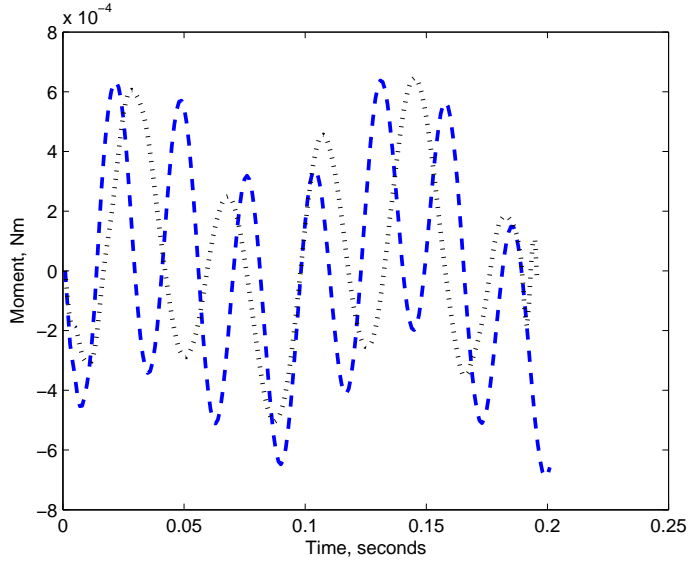

(b) $10 \mathrm{~Hz}$

Figure 19. Plunge motion: Comparison of root bending moment for Config 1 and Config 2 ; dashed line Config 1, dotted line - Config 2 


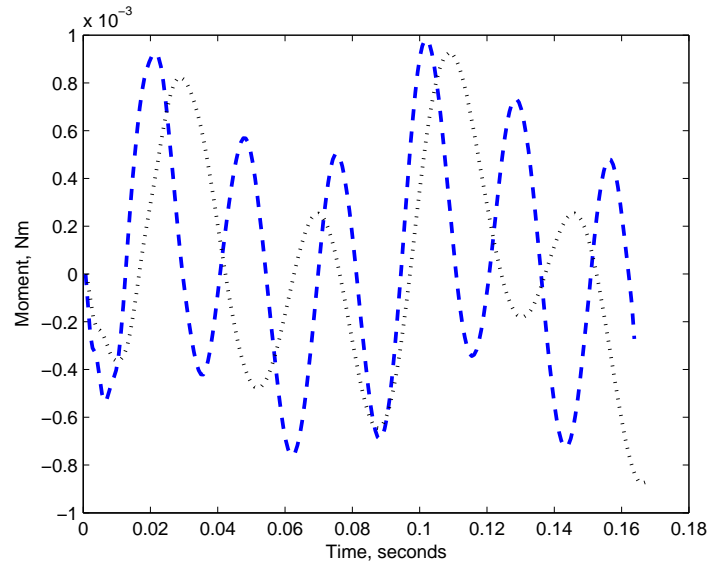

(a) $12 \mathrm{~Hz}$

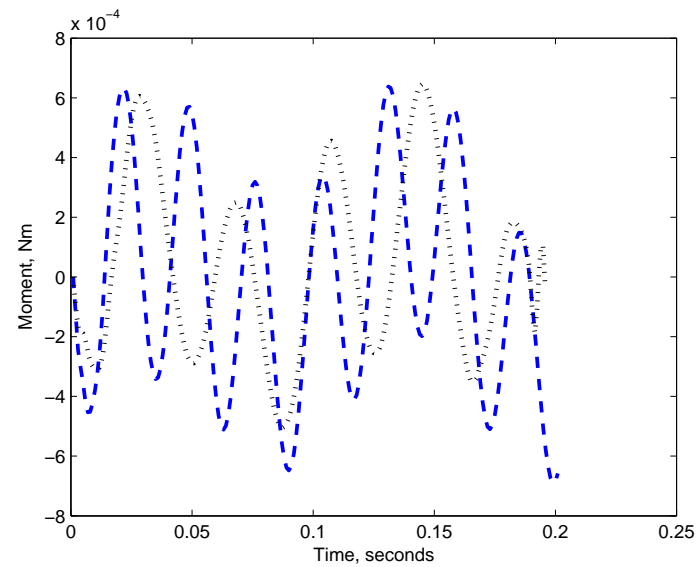

(b) $10 \mathrm{~Hz}$

Figure 20. Flap motion: Comparison of root bending moment for Config 1 and Config 2 ; dashed line - Config 1 , dotted line - Config 2 